\title{
Spectral properties of near-Earth and Mars-crossing asteroids using Sloan photometry
}

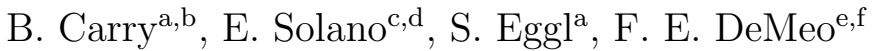 \\ ${ }^{a} I M C C E$, Observatoire de Paris, PSL Research University, CNRS, Sorbonne Uni-versit \\ es, UPMC Univ Paris 06, Univ. Lille \\ ${ }^{b}$ European Space Astronomy Centre, ESA, P.O. Box 78, 28691 Villanueva de la Cañada, \\ Madrid, Spain \\ ${ }^{c}$ Centro de Astrobiologia (INTA-CSIC), Departamento de Astrofisica. P.O. Box 78, \\ E-28691 Villanueva de la Cañada, Madrid, Spain \\ ${ }^{d}$ Spanish Virtual Observatory \\ ${ }^{e}$ Department of Earth, Atmospheric and Planetary Sciences, MIT, 77 Massachusetts \\ Avenue, Cambridge, MA, 02139, USA \\ ${ }^{f}$ Harvard-Smithsonian Center for Astrophysics, 60 Garden Street, MS-16, Cambridge, \\ MA, 02138, USA
}

\begin{abstract}
The nature and origin of the asteroids orbiting in near-Earth space, including those on a potentially hazardous trajectory, is of both scientific interest and practical importance. We aim here at determining the taxonomy of a large sample of near-Earth and Mars-crosser asteroids and analyze the distribution of these classes with orbit. We use this distribution to identify the source regions of near-Earth objects and to study the strength of planetary encounters to refresh asteroid surfaces. We measure the photometry of these asteroids over four filters at visible wavelengths on images taken by the Sloan Digital Sky Survey (SDSS). These colors are used to classify the asteroids into a taxonomy consistent with the widely used Bus-DeMeo taxonomy (DeMeo et al., Icarus 202, 2009) based on visible and near-infrared spectroscopy. We report
\end{abstract}

Email address: benoit.carry@oca.eu (B. Carry) 
here on the taxonomic classification of 206 near-Earth and 776 Mars-crosser asteroids determined from SDSS photometry, representing an increase of $40 \%$ and $663 \%$ of known taxonomy classifications in these populations. Using the source region mapper by Greenstreet et al. (Icarus, 217, 2012), we compare for the first time the taxonomic distribution among near-Earth and main-belt asteroids of similar diameters. Both distributions agree at the few percent level for the inner part of the Main Belt and we confirm this region as a main source of near-Earth objects. The effect of planetary encounters on asteroid surfaces are also studied by developing a simple model of forces acting on a surface grain during planetary encounter, which provides the minimum distance at which a close approach should occur to trigger resurfacing events. By integrating numerically the orbit of the 519 S-type and 46 Q-type asteroids in our sample back in time for 500,000 years and monitoring their encounter distance with Venus, Earth, Mars, and Jupiter, we seek to understand the conditions for resurfacing events. The population of Q-type is found to present statistically more encounters with Venus and the Earth than S-types, although both S- and Q-types present the same amount of encounters with Mars.

Keywords: Near-Earth objects, Asteroids, composition, Photometry

\section{Introduction}

Asteroids are the leftovers of the building blocks that accreted to form the planets in the early Solar System. They are also the progenitors of the constant influx of meteorites falling on the planets, including the Earth. Apart from the tiny sample of rock from asteroid (25143) Itokawa brought back by 
the Hayabusa spacecraft (Nakamura et al. 2011), these meteorites represent our sole possibility to study in details the composition of asteroids. Identifying their source regions is crucial to determine the physical conditions and abundances in elements that reigned in the protoplanetary nebula around the young Sun (see, e.g., McSween et al. 2006). From the analysis of a bolide trajectory, it is possible to reconstruct its heliocentric orbit and to find its parent body (e.g., Gounelle et al. 2006), but such determinations have been limited to a few objects only (Rudawska et al. 2012).

Among the different dynamical classes of asteroids, the near-Earth and Mars-crosser asteroids (NEAs and MCs), whose orbits cross that of the telluric planets, form a transient population. Their typical lifetime is of a few million years only (Bottke et al. 2002; Morbidelli et al. 2002) before being ejected from the Solar System, falling into the Sun, or impacting a planet. These populations are therefore constantly replenished by asteroids from the main asteroid belt, the largest reservoir of known small bodies, between Mars and Jupiter.

The resonances between the orbits of asteroids and that of Jupiter have been long thought (Wetherill 1979; Wisdom 1983) to provide the kick in eccentricity necessary to place asteroids on planet-crossing orbits. It was later found that the secular resonance $\nu_{6}$, delimiting the inner edge of the main belt, and the 3:1 mean-motion resonance (MMR) with Jupiter, separating the inner from the middle belt, were the most effective, compared to the 5:2 resonance, for instance, which tends to eject asteroids from the solar system (see Morbidelli et al. 2002, for a review). The major role played by the $\nu_{6}$ resonance was confirmed by the comparison between the reflectance spectra 
of the most common meteorites, the ordinary chondrites (OCs, $80 \%$ of all meteorite falls), the dominant class in the near-Earth space, the S-type asteroids (about $65 \%$ of the observed population, Binzel et al. 2004), and the dynamical family of S-types asteroids linked with (8) Flora in the inner belt (Vernazza et al. 2008).

The NEAs also represent ideal targets for space exploration owing to their close distance from Earth. This proximity is quantified by the energy required to set a spacecraft on a rendezvous trajectory and is often expressed as $\Delta v$ (in $\mathrm{km} / \mathrm{s}$ ), the required change in speed. This is the reason why the first mission to an asteroid targeted the Amor (433) Eros (Veverka et al. 2000), why all the targets of sample-return missions were selected among NEAs: (25143) Itokawa for JAXA Hayabusa (Fujiwara et al. 2006), (101955) Bennu for NASA OSIRIS-REx (Origins-Spectral Interpretation-Resource IdentificationSecurity-Regolith Explorer, Lauretta et al. 2011), (162 173) Ryugu for JAXA Hayabusa2 (Yano et al. 2010), and (175 706) 1996 FG3 and (341 843) 2008 EV5 for the former ESA M3/M4 candidate MarcoPolo-R (Barucci et al. 2012) and ARM (Asteroid Redirect Mission, Abell et al. 2015), and why the recent proposition for a demonstration project of an asteroid deflection by ESA, AIDA (Asteroid Impact \& Deflection Assessment, Murdoch et al. 2012), targets the NEA (65 803) Didymos. This latter point, the protection from asteroid hazard, is certainly the most famous aspect of the asteroid research known to the general public, and has triggered many initiatives leading to breakthroughs in NEA discovery and characterization of their surface and physical properties (see, e.g., Binzel 2000; Stokes et al. 2000; Ostro et al. 2002; Binzel et al. 2004; Jedicke et al. 2007; Mainzer et al. 2011a; Mueller et al. 
2011, among others).

In both attempting to link NEAs and MCs transient populations with their source regions and meteorites and designing a protection strategy, the study of their composition is key. Indeed, dynamical studies allows to determine relative probabilities of the origin of asteroids belonging to those populations (e.g., Bottke et al. 2002; Greenstreet et al. 2012). These links are however not sufficient, and must be ascertained by compositional similarities (Vernazza et al. 2008; Binzel et al. 2015; Reddy et al. 2015). Moreover, different compositions yield different densities and internal structure/cohesion (Carry 2012), and an asteroid on a impact trajectory with Earth of a given size will require a different energy to be deflected or destroyed according to its nature (Jutzi and Michel 2014).

Here, we aim at classifying a large number of near-Earth and Mars-crosser asteroids into broad compositional groups by using imaging archival data. We present in Section 2 the procedure we used to retrieve the photometry at visible wavelengths from the publicly available images of the Sloan Digital Sky Survey (SDSS). We describe in Section 3 how we use the SDSS photometry to classify the objects into the commonly-used Bus-DeMeo taxonomy of asteroids (DeMeo et al. 2009), following the work by DeMeo and Carry (2013). We present the results of the classification in Section 4 before discussing their implications for source regions in Section 5 and for surface rejuvenation processes in Section 6. 


\section{Visible photometry for the Sloan Digital Sky Survey}

\subsection{The Sloan Digital Sky Survey}

The Sloan Digital Sky Survey (SDSS) is a wide-field imaging survey dedicated to observing galaxies and quasars at different wavelengths. From 1998 to 2009 , the survey covered over 14,500 square degrees in 5 filters: $\mathrm{u}^{\prime}, \mathrm{g}^{\prime}, \mathrm{r}^{\prime}$, $\mathrm{i}^{\prime}, \mathrm{z}^{\prime}$ (centered on 355.1, 468.6, 616.5, 748.1 and $893.1 \mathrm{~nm}$ ), with estimated limiting magnitude of $22.0,22.2,22.2,21.3$, and 20.5 for $95 \%$ completeness (Ivezić et al. 2001).

\subsection{The Moving Object Catalog}

In the course of the survey, 471,569 moving objects were identified in the images and listed in the Moving Object Catalogue (SDSS MOC, currently in its 4th release, including observations through March 2007). Among these, 220,101 were successfully linked to 104,449 unique objects corresponding to known asteroids (Ivezić et al. 2001). The remaining 251,468 moving objects listed in the MOC corresponded to unknown asteroids at the time of the release (August 2008).

First, we keep objects assigned a number or a provisional designation only, i.e., those for which we can retrieve the orbital elements. Among these, we select the near-Earth and Mars-crossers asteroids according to the limits on their semi-major axis, perihelion, and aphelion listed in Table 1, resulting in 2071 observations of 1315 unique objects. We then remove observations that are deemed unreliable: with any apparent magnitudes greater than the limiting magnitudes reported above (Section 2.1), or any photometric uncertainty greater than 0.05 . These constraints remove a large portion of 


\begin{tabular}{|c|c|c|c|c|c|c|c|c|c|}
\hline \multirow[t]{2}{*}{ Class } & \multicolumn{2}{|c|}{$\mathrm{a}(\mathrm{au})$} & \multicolumn{2}{|c|}{$\mathrm{q}(\mathrm{au})$} & $\mathrm{Q}(\mathrm{au})$ & $\mathrm{MOC} 4$ & SVO-MOC & $\mathrm{SVO}_{\text {griz }}$ & $\mathrm{SVO}_{\text {gri }}$ \\
\hline & $\min$. & $\max$. & $\min$ & $\max$ & $\min$. & & & & \\
\hline Atens & - & 1.0 & - & $\mathrm{Q}_{\sigma^{\top}}$ & 0.983 & - & - & 10 & 1 \\
\hline Apollos & 1.0 & - & - & 1.017 & - & 14 & 18 & 82 & 70 \\
\hline Amors & - & - & 1.017 & 1.3 & - & 29 & 73 & 111 & 40 \\
\hline Mars-crosser & - & - & - & $\mathrm{Q}_{0^{7}}$ & - & 310 & 383 & 622 & 567 \\
\hline Total & - & - & - & - & - & 353 & 474 & 825 & 678 \\
\hline
\end{tabular}

Table 1: Definition of the dynamical classes of near-Earth and Mars-crosser asteroids used in present study, based on the semi-major axis (a), perihelion (q), and aphelion (Q) of their orbit. All the objects have a perihelion inward of Mars aphelion $\left(\mathrm{Q}_{0^{*}}\right)$ at $1.666 \mathrm{au}$. The number of objects listed in the SDSS MOC4 (Section 2.2), identified in SDSS MOC4 using SkyBoT (SVO-MOC, Section 2.3), and by the SVO NEA project (Section 2.4) are also listed. See Fig. 1 for the distribution of these objects in the orbital element space.

the dataset (about 75\%), primarily due to the larger typical error for the $\mathrm{z}^{\prime}$ filter. While there is only a small subset of the sample remaining, we are assured of the quality of the data (see DeMeo and Carry 2013, for additional information on the definition of photometric cuts). Additionally, for higher errors, the ambiguity among taxonomic classes possible for an object becomes so large that the classification (Section 3) becomes essentially meaningless. In this selection process, we kept 588 observations of 353 individual asteroids from the SDSS MOC4, as listed in Table 1.

\subsection{Identifying unknown objects in the MOC4}

As mentioned above, more than half of the MOC4 entries had not been linked with known asteroids. At the time of the release (August 2008), about 460,000 asteroids had been discovered and 350,000 were numbered 
(i.e., had well-constrained orbits allowing easy cross-matching with SDSS detected sources). The current number of discovered asteroids has now risen above 700,000, with more than 370,000 numbered objects. We therefore use the improved current knowledge on the asteroid population to link unknown MOC sources to known objects.

We use the Virtual Observatory (VO) SkyBoT cone-search service (Berthier et al. 2006), hosted at $\mathrm{IMCCE}^{1}$, for that purpose. SkyBoT pre-computes weekly the ephemeris of all known Solar System objects for the period 1889-2060, and stores their heliocentric positions with a time step of 10 days, allowing fast computation of positions at any time. The cone-search tool allows to request the list of known objects within a field of view at any given epoch as seen from Earth in typically less than $10 \mathrm{~s}$. We send 251,468 requests to SkyBoT, corresponding to the 251,468 unknown objects in the MOC4, centered on the MOC4 object's coordinates, at the reported epoch of observation, within a circular field of view of 30 arcseconds. Although many asteroids among the 700,000 known have position uncertainty larger than this value (as derived from their orbital parameter uncertainty), this cut ensures that we only keep objects with a high probability to be linked with the MOC sources.

To further restrict the number of false-positive associations, we compare the position, apparent motion, and apparent magnitude of the MOC sources to that predicted from ephemeris provided by SkyBoT, based on the database of orbital elements AstOrb² ${ }^{2}$. We consider successful association of SDSS

\footnotetext{
${ }^{1}$ http://vo.imcce.fr/webservices/skybot/

${ }^{2}$ http://asteroid.lowell.edu/
} 
sources with SkyBoT entry if the positions are closer than 30", the apparent V-Johnson magnitudes do not differ by more than 0.5 , and the apparent motions are co-linear (difference in $d \alpha \cos (\delta) / d t$ and $d \delta / d t$ of less than $3^{\prime \prime} / \mathrm{h}$ ). However, neither SkyBoT nor MOC4 provide estimates on the uncertainty in the apparent velocity. The only information is the uncertainty in the velocity components parallel and perpendicular to the SDSS scanning direction. The mean value of this error (both in the parallel and in the perpendicular direction) is of $1^{\prime \prime} / \mathrm{h}$. We are taking this value as one standard deviation to set the cut above.

Of the 251,468 unidentified MOC sources, SkyBoT provides known asteroids within 30 arcseconds for 68,497 (27\%), corresponding to 41,055 unique asteroids. We trim this value to 57,646 (36,730 asteroids) for which the association can be considered certain. The vast majority of these now-identified asteroids have orbits within the main belt (35,404, corresponding to $96 \%)$, but some are NEAs $(48,0.1 \%)$, or MCs $(73,0.2 \%)$. Their respective numbers are reported in Table 1. The complete list of MOC entries associated to known asteroids (277,747 entries associated to 141,388 asteroids) is freely accessible $^{3}$.

\subsection{The SVO Near-Earth Asteroids Recovery Program}

In addition, we search the images of the SDSS for NEAs and MCs that were either not identified as moving objects by the automatic SDSS pipeline, rejected by the $\mathrm{MOC}$ data selection ${ }^{4}$, or imaged after the latest compilation

\footnotetext{
${ }^{3}$ http://svo2.cab.inta-csic.es/vocats/svomoc

${ }^{4}$ http://www.astro.washington.edu/users/ivezic/sdssmoc/sdssmoc.html
} 
of the SDSS MOC4 (i.e., observed after March 2007). Indeed, only moving objects with an apparent motion between 0.05 and $0.050 \mathrm{deg} /$ day were included in the MOC, leaving a significant fraction of NEAs un-cataloged (Solano et al. 2013).

We use the resources of the citizen-science project "Near-Earth Asteroids Recovery Program" of the Spanish Virtual Observatory (SVO) which was originally designed for this very purpose: to identify and measure the astrometry of NEAs in archival imaging data (Solano et al. 2013). For each Aten, Amor, Apollo, and Mars-crosser listed by the Minor Planet Center ${ }^{5}$ (MPC), its ephemeris are computed over the period of operation of the SDSS imaging survey (1998 to 2009) and compared to the footprints of the images of the survey. The images possibly containing an object brighter than the SDSS limiting magnitude $(\mathrm{V}=22)$ are then proposed to the public for identification through a web portal ${ }^{6}$. Since the beginning of the project in 2011, over 2,500 astrometry measurements of about 600 NEAs not identified in the MOC have been reported to the MPC (see Solano et al. 2013, for details on the project).

To compute the photometry of the NEAs measured by the users we first searched in the photometric catalog of the 8th SDSS Data Release ${ }^{7}$. If no photometry associated with the NEA was found, we ran SExtractor on the corresponding images and calibrated the SExtractor magnitudes by comparing them with the SDSS magnitudes of the sources identified in the image.

\footnotetext{
${ }^{5}$ http://minorplanetcenter.org/

${ }^{6}$ http://www.laeff.cab.inta-csic.es/projects/near/main/

${ }^{7}$ http://cdsarc.u-strasbg.fr/viz-bin/Cat?II/306
} 
Owing to the more stringent limiting magnitude in the $z^{\prime}$ filter, many asteroids are identified over three bands $\left(\mathrm{g}^{\prime} \mathrm{r}^{\prime} \mathrm{i}^{\prime}\right)$ only. We also report these objects here, although deriving a taxonomic classification is of course less accurate. Overall, we collect 1194 four bands $\left(\mathrm{g}^{\prime} \mathrm{r}^{\prime} \mathrm{i}^{\prime} \mathrm{z}^{\prime}\right)$ photometry measurements of 825 unique asteroids and 976 three-bands $\left(\mathrm{g}^{\prime} \mathrm{r}^{\prime} \mathrm{i}^{\prime}\right)$ photometry measurements of 678 distinct asteroids (Table 1). We present in Fig. 2 a comparison of our measurements with the magnitudes reported in the SDSS MOC4 for the common asteroids in both sets, showing excellent agreement (values agree with a standard deviation of $0.05 \mathrm{mag}$ ).

\section{Taxonomic classification}

The SDSS photometry has been used to classify asteroids according to their colors by many authors (e.g., Ivezić et al. 2002; Nesvorný et al. 2005; Parker et al. 2008; Carvano et al. 2010). One key advantage of the survey was the almost simultaneous acquisition of all filters $(5 \mathrm{~min}$ in total), hence limiting the impact of geometry-related lightcurve on the apparent magnitude. Here we follow the work by DeMeo and Carry $(2013,2014)$ in which the class definitions are set to be as consistent as possible with previous spectral taxonomies based on higher spectral resolution and larger wavelength coverage data sets, specifically Bus and Bus-DeMeo taxonomies (Bus and Binzel 2002; DeMeo et al. 2009). We present concisely the classification scheme below and refer to DeMeo and Carry (2013) for a complete description. 


\subsection{From SDSS to Bus-DeMeo taxonomy}

First, we convert the photometry into reflectance (using solar colors from Holmberg et al. 2006) and normalize them to unity in filter $\mathrm{g}^{\prime}$. Second, we compute the slope of the continuum over the $\mathrm{g}^{\prime}, \mathrm{r}^{\prime}$, and $\mathrm{i}^{\prime}$ filters (hereafter grislope), and the $\mathrm{z}^{\prime}-\mathrm{i}^{\prime}$ color (hereafter zi-color), representing the band depth of a potential $1 \mu \mathrm{m}$ band, because they are the most characteristic spectral distinguishers in all major taxonomies (beginning with Chapman et al. 1975). The classification into the taxonomy is then based on these two parameters.

As a results of the limited spectral resolution and range of SDSS photometry, we group together certain classes into broader complexes (see correspondences in Table 2). For asteroids with multiple observations that fall under multiple classifications, we use the tree-like selection to assign a final class (see DeMeo and Carry (2013) for details and DeMeo et al. (2014a) for an example of a spectroscopic confirmation campaign of the SDSS classification used here). We successfully classify 982 asteroids from the sample of 1015 near-Earth and Mars-crosser asteroids with four-bands photometry (i.e., $97 \%$ of the sample).

For objects with three-bands photometry only, we set their $z^{\prime}$ magnitude to the limiting magnitude of 20.5 (Ivezić et al. 2001) as an upper limit for their brightness. We then classify these asteroids using the scheme presented above. Because the magnitude of 20.5 in $\mathrm{z}^{\prime}$ is an upper limit, the actual zi-color for these asteroids may be overestimated. The classification can therefore be degenerated, all the classes with similar gri-slope and lower zicolor being possible. We assign tentative classification to 254 asteroids from the sample of 678 near-Earth and Mars-crosser asteroids with three-bands 
photometry (i.e., $37 \%$ of the sample).

In all cases, we mark objects with peculiar spectral behavior with the historical notation "U" (for unclassified), and discard them from the analysis. There are 33 and 424 asteroids in the four-bands and three-bands photometry samples respectively for which we cannot assign a class. These figures highlight the ambiguity raised by the lack of information on the presence or absence of an absorption band around one micron, to which the $\mathrm{z}^{\prime}$ filter is sensitive.

\subsection{Gathering classifications from past studies}

Many different authors have reported on the taxonomic classification of NEAs. We gather here the results of Dandy et al. (2003), Binzel et al. (2004), Lazzarin et al. (2005), de León et al. (2006), de León et al. (2010), Thomas and Binzel (2010), Popescu et al. (2011), Ye (2011), Reddy et al. (2011), Polishook et al. (2012), Sanchez et al. (2013), and DeMeo et al. (2014b). These authors used different taxonomic schemes to classify their observations, using either broad-band filter photometry or spectroscopy, at visible wavelengths only or also in the near infrared. We therefore transpose the classes of these different schemes (Tholen 1984; Tholen and Barucci 1989; Bus and Binzel 2002; DeMeo et al. 2009) into the single, consistent, set of 10 classes we already use for the SDSS data. Here also, we attribute the historical "U" designation for objects with apparently contradictory classifications (e.g., QX or STD in Ye 2011). These pathological cases represent $15 \%$ of the objects with multiple class determinations. In total, we gather 1022 classifications for 648 objects listed in the literature. 


\section{Results}

We list in Table B.1 the photometry and the taxonomy of all 982 nearEarth and Mars-crossers asteroids with four-bands photometry. The 254 asteroids with three-bands photometry are listed separately in Table B.2, because their taxonomic classification is less robust. In many cases, the upper limit of 20.5 for their $\mathrm{z}^{\prime}$ magnitude provides a weak constraint on their taxonomy, and classes with high zi-color (mainly V-types) are more easily identified. This sample based on three-bands photometry only is therefore biased, but it can be used as a guideline for selecting targets for spectroscopic follow-ups. We concentrate below on the sample based on four-bands photometry.

The 206 NEAs presented here have absolute magnitudes between 12 and 23. Our sample fully overlaps with the size range of the 523 NEAs characterized by visible/near-infrared spectroscopy published to date and represents an increase of $\approx 40 \%$ of the current sample size (Fig. 3). A significant fraction $(46 \%)$ of the NEA population with $\mathrm{H}<16$ (about $2 \mathrm{~km}$ diameter for an albedo of 0.20 ) has a taxonomic classification. For smaller diameters, the fraction drops quickly to $10 \%$ and less. The sub-kilometer population of NEAs is therefore still poorly categorized.

The absolute magnitude of the 776 MCs reported here ranges from 11 to 19. Our sample represents the first classification of sub-kilometric Marscrossers, and a sixfold increase to the sample of $117 \mathrm{MCs}$ from spectroscopy (Fig. 3). Similarly to NEAs, about $40 \%$ of the MC population with $\mathrm{H}<14$ (about $5 \mathrm{~km}$ diameter for an albedo of 0.20 ) now has a taxonomic classification, and the fraction drops quickly to $10 \%$ for smaller diameters. 


\subsection{Taxonomy and orbital classes}

We present in Fig. 4 how the different classes distribute among the orbital populations. As already reported by Binzel et al. (2004), the broad S(including Q-types), C-, X-complexes, and V-types dominate the NEA population, the minor classes (A, B, D, K, L) accounting for a few percents only, similarly to what is found in the inner main belt (DeMeo and Carry 2013, 2014). We find that the S-complex encompasses twice as many objects as the $\mathrm{C}$ - and $\mathrm{X}$-complexes, compared to the threefold difference reported by Binzel et al. (2004).

The distribution of taxonomic classes among MCs is similar to that of Apollos and Amors (with only 8 Atens, our sample suffers from low-number statistics). Our findings of V-types accounting for roughly $5 \%$ of all MCs may therefore seem puzzling considering the lack of V-types among the

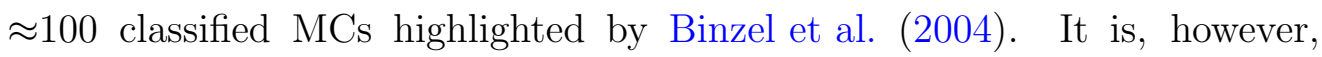
an observation selection effect. Indeed, Mars-crossers are inner-main belt (IMB) asteroids which eccentricity has been increased by numerous weak mean-motion resonances ("chaotic diffusion", see Morbidelli and Nesvorný 1999). The IMB hosting the largest reservoir of V-types in the solar system (Vestoids, Binzel and Xu 1993), V-types were expected in MC space.

The size distribution of known Vestoids however peaks at $\mathrm{H}=16$ (about $1.5 \mathrm{~km}$ diameter), and the largest members have an absolute magnitude of 14 (we use the list of Vestoids from Nesvorny 2012). All the V-types identified here have an absolute magnitude above 14, and so does (31 415) 1999 AK23 $(\mathrm{H}=14.4)$, the first $\mathrm{V}$-type among MCs reported recently by Ribeiro et al. (2014). Only 33 MCs had been characterized with this absolute magnitude 
or higher to date, and the previous lack of report of V-types among MCs is consistent with our findings.

\subsection{Low- $\delta v$ as space mission targets}

Within the 206 NEA serendipitously observed by the SDSS, we identify 36 potential targets for space missions based on their accessibility. We select all the NEA with a $\delta v$ below $6.5 \mathrm{~km} / \mathrm{s}$. As a matter of comparison, the required $\delta v$ to reach the Moon and Mars are of 6.0 and $6.3 \mathrm{~km} / \mathrm{s}$ (e.g., Abell et al. 2012). We list in Table 3 the basic characteristics of these potential targets, together with the targets already, or planned to be, visited by spacecraft.

Among the list of low- $\delta v$ objects, we find a large majority of S-types, following their dominance in the sample presented here of about $60 \%$. We, however, note the presence of potential D-, L-, and K-types. To date, of the 24 taxonomic classes, only C- (Mathilde, Ceres), S- (Ida, Eros, Gaspra, Itokawa, and Toutatis), Xe- (Steins), Xk- (Lutetia), and V-types (Vesta) have been visited by spacecraft. These potential D-, L-, and K-types targets may represent good opportunities for exploration. Data in the visible can only suggest the presence of an absorption band at $1 \mu \mathrm{m}$, and near-infrared data is required to confirm these potential classifications.

\section{Source regions}

The population of NEAs being eroded on short timescale $(<10 \mathrm{My})$ by planetary collisions and dynamical ejections, new objects must be injected in the NEA space to explain the current observed population. We use our sample of 982 NEAs and MCs to identify possible source regions. For that, 
we use the source region mapper ${ }^{8}$ by Greenstreet et al. (2012), built on the result of numerical simulations of the orbital evolution of test particules in the five regions defined by Bottke et al. (2002): $\nu_{6}$ secular resonance, 3:1 mean-motion resonance with Jupiter, Mars-crossers (MC), Outer Belt (OB), and Jupiter Family Comet (JFC).

For each object, we compute its probability $\mathcal{P}_{i}$ to originate from the $i^{\text {th }}$ source region. We then normalize all $\mathcal{P}_{i}$ for each source region. The sum of the normalized $\mathcal{P}_{i}$ over a given taxonomic class therefore represents the fraction of objects (by number) of this class in the source region (Table 4). Uncertainties are computed from the source region mapper uncertainties, quadratically added with the margin of error (at 95\% confidence level) to account for the sample size. We can then compare these predicted fractions to the observed distribution of taxonomic classes for each source region.

The vast majority of NEAs with taxonomic classification sustain diameters of less than $5 \mathrm{~km}$ (absolute magnitude above 14). Because the distribution of taxonomic classes in the main belt varies with diameter (DeMeo and Carry 2014), we need to compare the predicted fraction of Table 4 with objects with $\mathrm{H}>14$ in each source region. For the first time, thanks to the large dataset of asteroids provided by the Sloan Digital Sky Survey (SDSS), such information is available for the inner part of the main belt (DeMeo and Carry 2013, 2014) and is reported in Table 4 (3 to $5 \mathrm{~km}$ diameter range).

The comparison of the $\nu_{6}$ and 3:1 source regions (delimiting the inner belt) with the observations in Fig. 5 shows a very good match of the distributions

\footnotetext{
${ }^{8}$ Updated by S. Greenstreet from the original mapper to include the probability of the source regions of the MCs themselves.
} 
(correlation coefficient of 0.99 , maximum difference of $5 \%$ ). This validates the dynamical path from the inner belt to the near-Earth space as described in the model by Greenstreet et al. (2012). Although the inner belt is widely accepted as a major source for NEAs and meteorites (e.g., Bottke et al. 2002; Binzel et al. 2004; Vernazza et al. 2008; Binzel et al. 2015), the relative contribution of the different source regions particularly with respect to asteroid size is still a matter of debate. For the first time, we compare here populations of the same size range.

Unfortunately, there is no similar data set for the other source regions (MC, OB, and JFC) to be compared with our prediction. We can still note the overall trend of increasing fraction of C/D/X-types in OB and JFC compared with the inner regions, as expected. The fraction of K-types peaks in the outer belt, place of the Eos family, also as expected, although a steady distribution of K-types across source regions is also consistent within uncertainties. We also note a strong correlation between MC and small inner-belt asteroid populations (correlation coefficient 0.97 ), which is not related to the origin of NEAs, but of MC themselves, via chaotic diffusion from the inner belt (see Morbidelli and Nesvorný 1999; Michel et al. 2000).

A peculiar feature of Table 4 is the high fraction of S-type in all source regions. Based on present set of NEAs and the dynamical model by Greenstreet et al. (2012), one asteroid below $5 \mathrm{~km}$ diameter out of four should be an S-type in the outer belt and the same applies to Jupiter family comets. Although the census of composition in this size range $(3-5 \mathrm{~km})$ is far from being complete in these regions, there is a bias toward detecting S-types due to their high albedo $(\approx 0.20)$ compared to that of the $\mathrm{C} / \mathrm{P} / \mathrm{D}$-types found there (around 
0.05, see Mainzer et al. 2011b; DeMeo and Carry 2013, for albedo averages over taxonomic classes) S-types are minor contributors to the outer belt for diameters above $5 \mathrm{~km}$ (DeMeo and Carry 2014) and searches for S-type material have been unsuccessful among Cybeles, Hildas, and Trojans (see Emery and Brown 2003, 2004; Emery et al. 2011; Fornasier et al. 2004, 2007; Yang and Jewitt 2007, 2011; Roig et al. 2008; Gil-Hutton and Brunini 2008; Marsset et al. 2014). However, only $4 \%$ of the sample (42 objects) are predicted to originate from the $\mathrm{OB}$ and JFC source regions. The results for these regions is, thus, based on small number statistics. The large fraction of S-types in these source regions suggests nevertheless that dynamical models may require further refinements. 


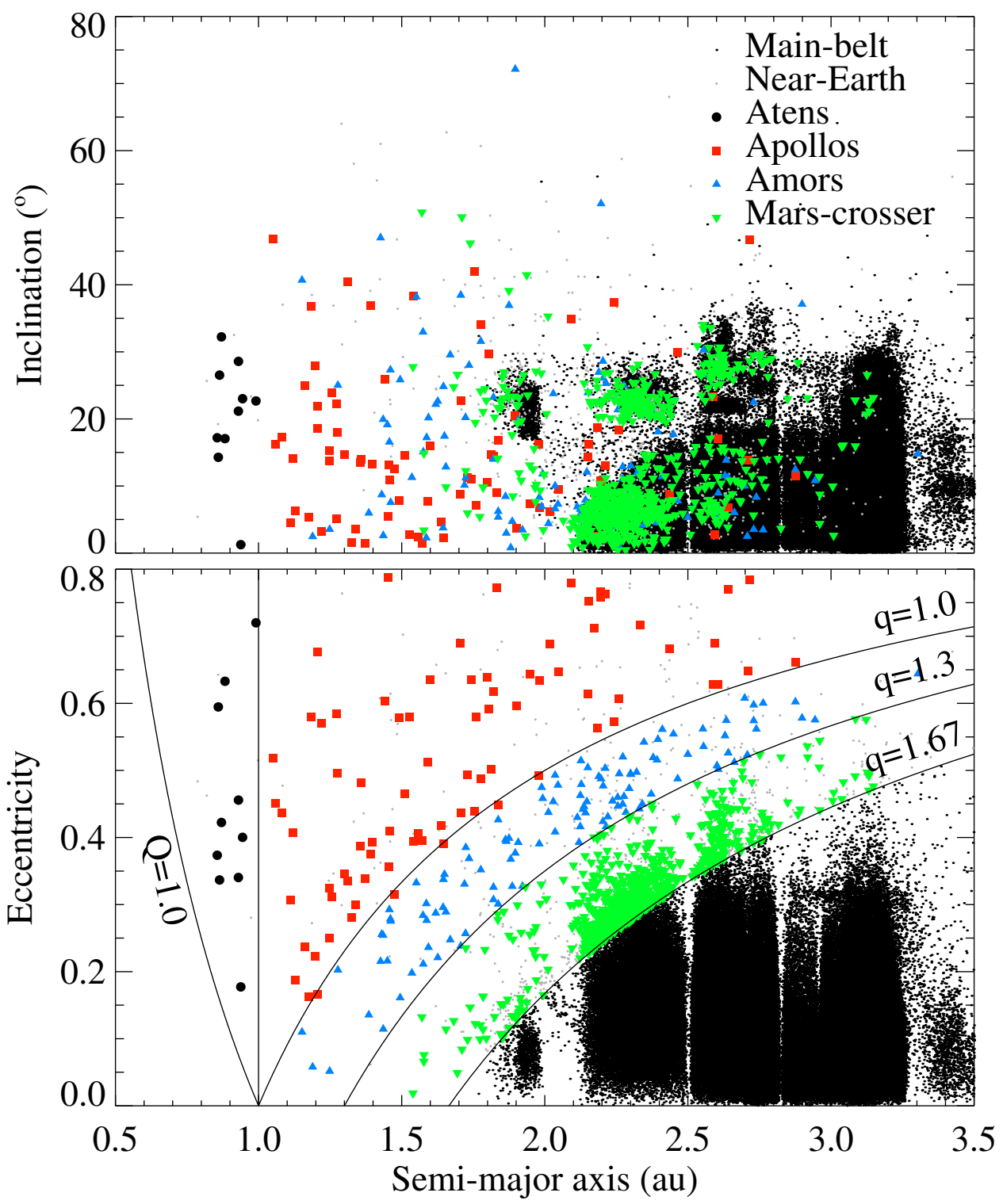

Figure 1: Distribution of the NEAs and Mars-crossers studied here as a function of their osculating elements (semi-major axis, eccentricity, and inclination). The black dots represent the first 10,000 main-belt asteroids, the grey dots the 532 NEAs with spectral classification from the literature (see Section 3.2), and the black circles, red squares, blue triangle, and green stars the Atens, Apollos, Amors, and Mars-crossers we classify from SDSS. 


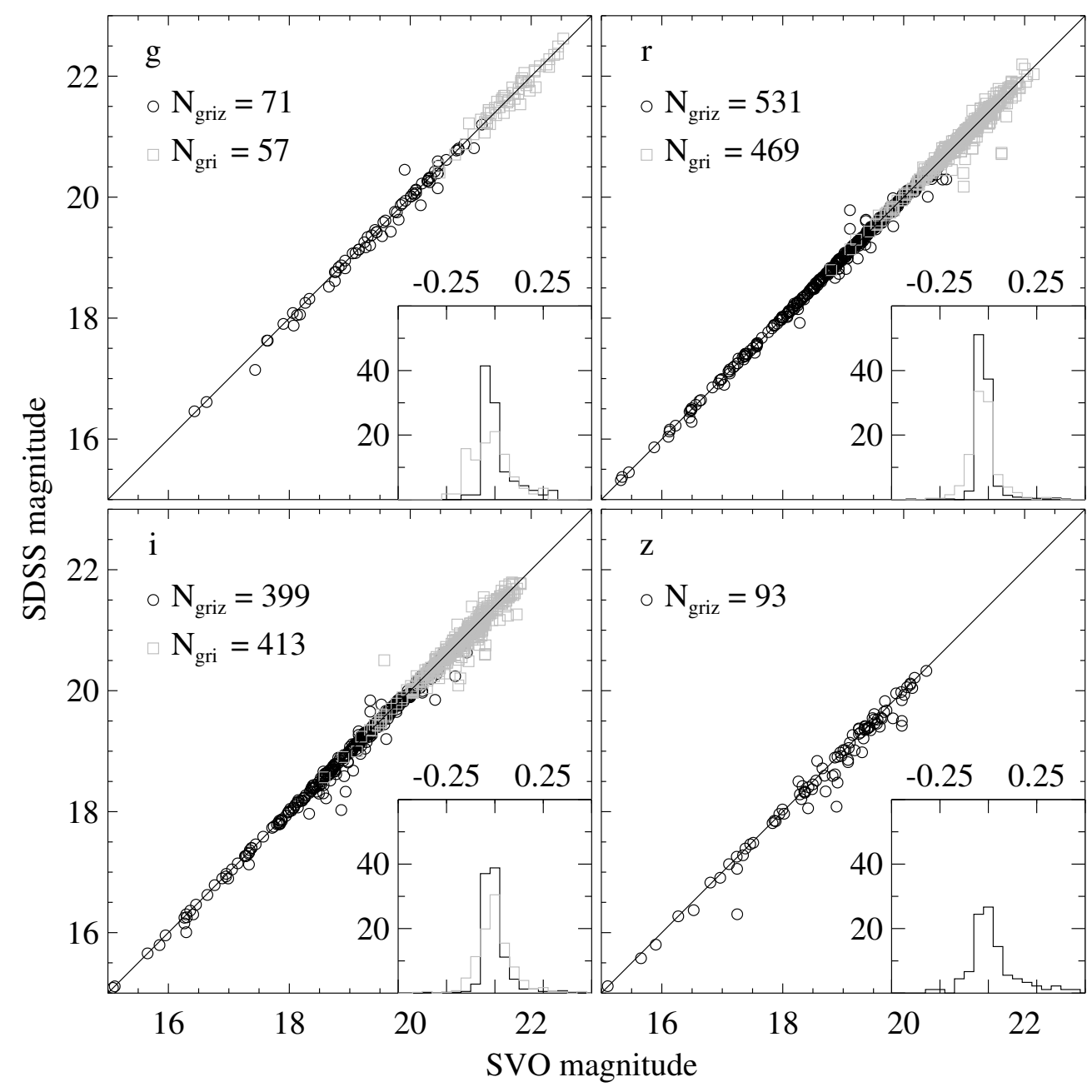

Figure 2: Comparison of the apparent magnitude measured by our system (SVO magnitude) with that reported in the SDSS MOC4 (SDSS magnitude) for the four $\mathrm{g}^{\prime}, \mathrm{r}^{\prime}, \mathrm{i}^{\prime}$, and $\mathrm{z}^{\prime}$ filters. For each, the number of common objects is reported, for both four-bands (black circles) and three-bands (grey squares) sets. The inserted histograms show the distribution of the magnitude difference (MOC-SVO) 


\begin{tabular}{|c|c|}
\hline SDSS \& Literature & Bus-DeMeo \\
\hline A, AR, AS & $\mathrm{A}$ \\
\hline B & $\mathrm{B}$ \\
\hline $\mathrm{C}, \mathrm{C}: \mathrm{Cb}, \mathrm{Cg}, \mathrm{Ch}$ & $\mathrm{C}$ \\
\hline $\mathrm{D}, \mathrm{DT}$ & $\mathrm{D}$ \\
\hline $\mathbf{K}, \mathrm{K}:$ & $\mathrm{K}$ \\
\hline $\mathbf{L}, \mathbf{L d}$ & $\mathrm{L}$ \\
\hline $\mathrm{Q}, \mathrm{Q} / \mathrm{R}, \mathrm{R}, \mathrm{RQ}$ & $\mathrm{Q}$ \\
\hline $\mathrm{O}, \mathrm{Q} / \mathrm{R} / \mathrm{S}, \mathrm{R}, \mathrm{RS}$ & $\mathrm{S}$ \\
\hline S, S:, S(I $\rightarrow$ V), Sa, Sk, Sl, Sq, Sq:, Sr & $\mathrm{S}$ \\
\hline $\mathbf{V}, \mathrm{V}:$ & $\mathrm{V}$ \\
\hline E, M, P, X, X: XT, Xc, Xe, Xk & $\mathrm{X}$ \\
\hline $\mathrm{U}, \mathrm{C}(\mathrm{u}), \mathrm{R}(\mathrm{u}), \mathrm{S}(\mathrm{u}), \mathrm{ST}, \mathrm{STD}, \mathrm{QX}$ & $\mathrm{U}$ \\
\hline
\end{tabular}

Table 2: Correspondence between the classes from different taxonomies (e.g., Tholen and Barucci 1989; Bus and Binzel 2002) found in the literature, used to classify the SDSS photometry (in bold), and their equivalence in the reduced version of the taxonomy by DeMeo et al. (2009) adopted here, following the work by DeMeo and Carry (2013). We strive to preserve the most extreme classes (like A, B) and we convert the tentative classification (e.g., RS) into their broader, safer, complex (S). We also consider as unknown (U) any data of insufficient quality. 


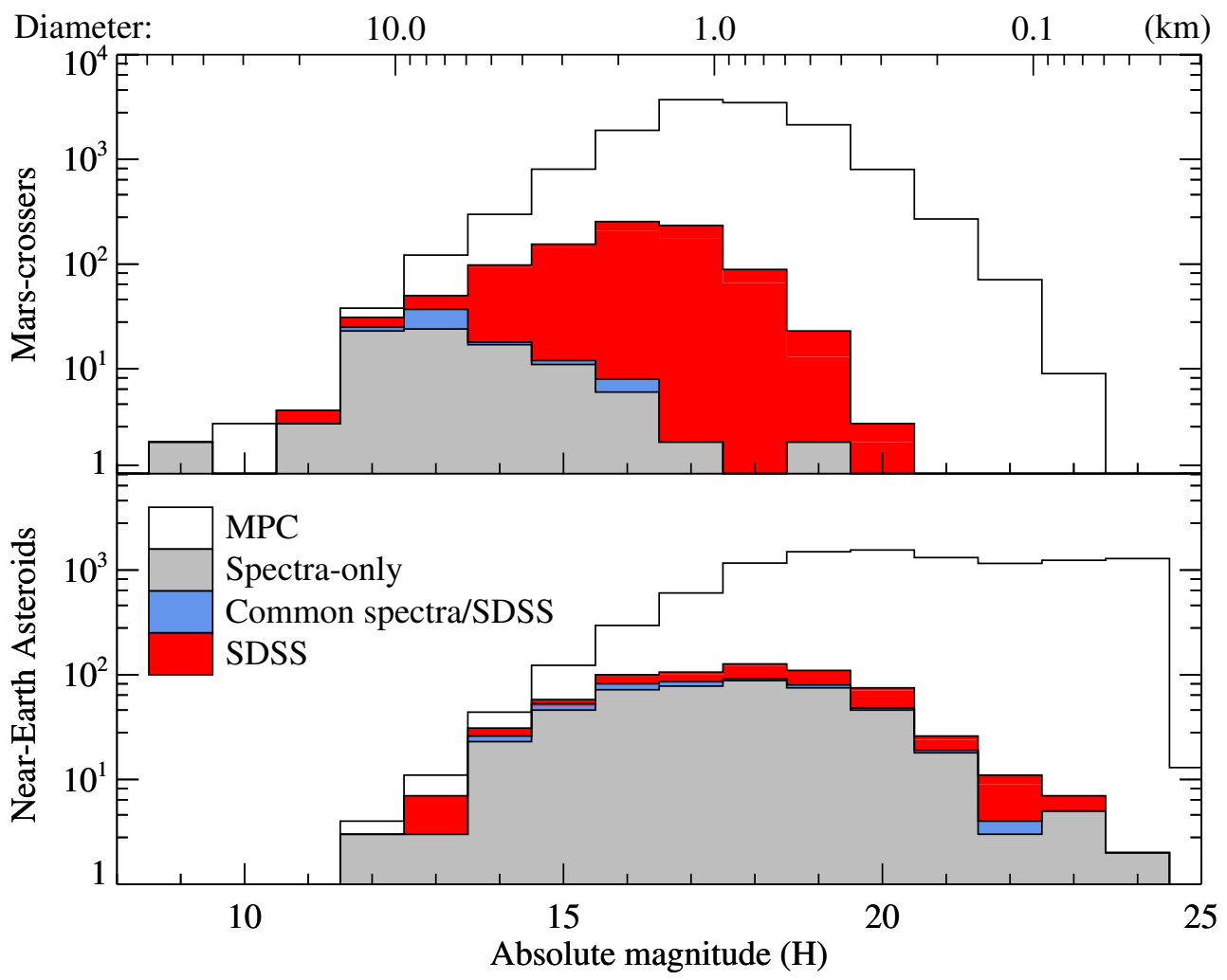

Figure 3: Distribution of the known taxonomy for near-Earth and Mars-crosser asteroids as a function of their absolute magnitude $\mathrm{H}$. The white histogram corresponds to the total number of discoveries (listed in AstOrb), the grey to the taxonomic classification found in the literature, the blue to the overlap between literature and present study, and the red the four-bands set. An approximative conversion to diameter is also reported, using $D=1329 \times p_{v}^{-0.5} \times 10^{-0.2 H}$, with the geometric albedo $p_{v}$ taken as 0.2 (the majority, $\approx 60 \%$, of the objects classified here being S-types). 

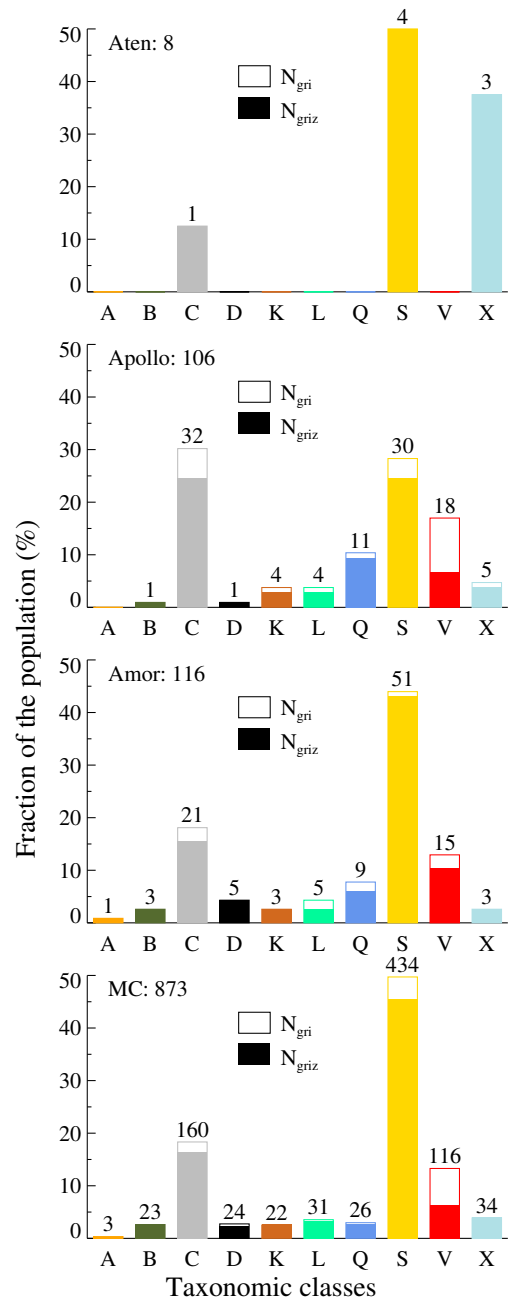

Figure 4: Distribution of the taxonomic classes for each dynamical group: Aten, Apollo, Amor, and Mars-crosser. The number of object with four-band photometry in each class is reported. Empty and filled bars stands for the three and four band photometry samples respectively. 


\begin{tabular}{|c|c|c|c|c|c|c|c|c|c|}
\hline Designation & Type & Class & $\delta v(\mathrm{~km} / \mathrm{s})$ & $\mathrm{H}$ & $s(\% / 100 \mathrm{~nm})$ & $z-\mathrm{i}$ & $\mathrm{a}(\mathrm{au})$ & $\mathrm{e}$ & $\mathrm{i}\left({ }^{\circ}\right)$ \\
\hline 2004 EU22 & $\mathrm{X}$ & Apollo & 4.420 & 23.00 & 0.78 & 0.044 & 1.175 & 0.162 & 5.3 \\
\hline 1996 XB27 & $\mathrm{D}$ & Amor & 4.750 & 22.00 & 0.85 & 0.123 & 1.189 & 0.058 & 2.5 \\
\hline 2000 TL1 & $\mathrm{C}$ & Apollo & 4.870 & 22.00 & -0.09 & 0.002 & 1.338 & 0.300 & 3.6 \\
\hline 2001 QC34 & Q & Apollo & 4.970 & 20.00 & 0.69 & -0.228 & 1.128 & 0.187 & 6.2 \\
\hline 1999 FN19 & S & Apollo & 5.020 & 22.00 & 0.96 & -0.135 & 1.646 & 0.391 & 2.3 \\
\hline 2000 SL10 & Q & Apollo & 5.080 & 21.00 & 0.86 & -0.189 & 1.372 & 0.339 & 1.5 \\
\hline $1994 \mathrm{CN} 2$ & $\mathrm{~S}$ & Apollo & 5.150 & 16.00 & 1.12 & -0.073 & 1.573 & 0.395 & 1.4 \\
\hline 2002 LJ3 & $\mathrm{S}$ & Amor & 5.280 & 18.00 & 1.07 & -0.258 & 1.462 & 0.275 & 7.6 \\
\hline 2004 UR & $\mathrm{C}$ & Apollo & 5.320 & 22.00 & 0.13 & -0.025 & 1.559 & 0.406 & 2.4 \\
\hline 2006 UP & $\mathrm{S}$ & Amor & 5.350 & 23.00 & 1.43 & -0.074 & 1.586 & 0.301 & 2.3 \\
\hline $1994 \mathrm{CC}$ & $\mathrm{S}$ & Apollo & 5.370 & 17.00 & 0.99 & -0.210 & 1.638 & 0.417 & 4.7 \\
\hline 2010 WY8 & K & Amor & 5.670 & 21.00 & 0.92 & -0.042 & 1.385 & 0.136 & 6.0 \\
\hline 2002 XP40 & $\mathrm{S}$ & Amor & 5.720 & 19.00 & 1.63 & -0.092 & 1.645 & 0.296 & 3.8 \\
\hline $1993 \mathrm{QA}$ & $\mathrm{D}$ & Apollo & 5.740 & 18.00 & 1.02 & 0.174 & 1.476 & 0.315 & 12.6 \\
\hline 2004 RK9 & $\mathrm{C}$ & Amor & 5.760 & 21.00 & 0.14 & -0.050 & 1.837 & 0.426 & 6.2 \\
\hline $2001 \mathrm{FC7}$ & $\mathrm{C}$ & Amor & 5.780 & 18.00 & 0.16 & -0.034 & 1.436 & 0.115 & 2.6 \\
\hline $1977 \mathrm{VA}$ & $\mathrm{C}$ & Amor & 5.940 & 19.20 & 0.34 & -0.010 & 1.866 & 0.394 & 3.0 \\
\hline 2001 WL15 & $\mathrm{C}$ & Amor & 6.000 & 18.00 & 0.34 & -0.179 & 1.989 & 0.475 & 6.9 \\
\hline 2000 XK44 & $\mathrm{L}$ & Amor & 6.080 & 18.00 & 1.24 & 0.020 & 1.724 & 0.385 & 11.2 \\
\hline $2003 \mathrm{BH}$ & $\mathrm{V}$ & Apollo & 6.090 & 20.00 & 1.79 & -0.273 & 1.456 & 0.356 & 13.1 \\
\hline 2000 NG11 & $\mathrm{X}$ & Amor & 6.130 & 17.00 & 0.27 & 0.089 & 1.881 & 0.368 & 0.8 \\
\hline 2000 RW37 & $\mathrm{C}$ & Apollo & 6.150 & 20.00 & 0.45 & -0.166 & 1.248 & 0.250 & 13.8 \\
\hline 2001 FD90 & $\mathrm{V}$ & Amor & 6.200 & 19.00 & 0.75 & -0.419 & 2.046 & 0.478 & 7.3 \\
\hline 2002 PG80 & $\mathrm{S}$ & Amor & 6.210 & 18.00 & 1.09 & -0.225 & 2.013 & 0.438 & 4.4 \\
\hline $2004 \mathrm{VB}$ & $\mathrm{S}$ & Apollo & 6.260 & 20.00 & 1.04 & -0.200 & 1.458 & 0.409 & 10.9 \\
\hline 1993 DQ1 & $\mathrm{S}$ & Amor & 6.278 & 16.00 & 1.20 & -0.207 & 2.036 & 0.493 & 10.0 \\
\hline 2000 YG4 & $\mathrm{Q}$ & Amor & 6.300 & 20.00 & 0.61 & -0.155 & 2.211 & 0.503 & 2.6 \\
\hline 2004 KD1 & $\mathrm{C}$ & Amor & 6.330 & 17.00 & 0.13 & -0.110 & 1.720 & 0.331 & 10.1 \\
\hline 2004 RS25 & $\mathrm{C}$ & Amor & 6.410 & 20.00 & 0.39 & -0.057 & 2.128 & 0.479 & 6.7 \\
\hline 2004 QZ2 & $\mathrm{S}$ & Amor & 6.470 & 18.00 & -5.74 & -0.227 & 2.260 & 0.495 & 1.0 \\
\hline
\end{tabular}




\begin{tabular}{c|rrrcr|rr}
\hline \hline \multirow{2}{*}{ Class } & \multicolumn{5}{|c|}{ Source regions } & \multicolumn{2}{c}{ IMB $_{3-5 \mathrm{~km}}$} \\
& \multicolumn{1}{|c}{$\mathrm{MC}$} & \multicolumn{1}{c}{$\nu_{6}$} & \multicolumn{1}{c}{$\mathrm{MMR}_{3: 1}$} & \multicolumn{1}{c}{$\mathrm{OB}$} & \multicolumn{1}{c}{$\mathrm{JFC}$} & $(\#)$ & $(\%)$ \\
\hline $\mathrm{A}$ & $0.4 \pm 5.9$ & $0.5 \pm 6.3$ & $0.3 \pm 5.5$ & $0.0 \pm 3.0$ & $0.0 \pm 3.0$ & 9 & 0.4 \\
$\mathrm{~B}$ & $2.4 \pm 5.8$ & $1.7 \pm 5.4$ & $2.9 \pm 6.1$ & $6.1 \pm 7.5$ & $0.4 \pm 4.3$ & 34 & 1.5 \\
$\mathrm{C}$ & $15.7 \pm 5.2$ & $20.5 \pm 5.4$ & $22.7 \pm 5.6$ & $38.6 \pm 6.6$ & $47.3 \pm 10.6$ & 589 & 25.7 \\
$\mathrm{D}$ & $2.5 \pm 5.7$ & $2.3 \pm 5.6$ & $2.5 \pm 5.8$ & $5.8 \pm 7.2$ & $9.7 \pm 8.5$ & 20 & 0.9 \\
$\mathrm{~K}$ & $2.8 \pm 5.9$ & $2.5 \pm 5.8$ & $2.4 \pm 5.7$ & $4.8 \pm 6.9$ & $1.8 \pm 7.3$ & 57 & 2.5 \\
$\mathrm{~L}$ & $3.8 \pm 5.9$ & $3.4 \pm 5.7$ & $4.4 \pm 6.1$ & $1.9 \pm 5.1$ & $0.4 \pm 4.2$ & 73 & 3.2 \\
$\mathrm{Q}$ & $3.6 \pm 5.6$ & $4.3 \pm 5.8$ & $6.3 \pm 6.4$ & $3.8 \pm 5.8$ & $2.4 \pm 5.5$ & 0 & 0.0 \\
$\mathrm{~S}$ & $52.8 \pm 4.6$ & $44.5 \pm 4.6$ & $42.0 \pm 4.8$ & $23.8 \pm 4.8$ & $23.4 \pm 6.9$ & 1145 & 50.0 \\
$\mathrm{~V}$ & $13.0 \pm 5.5$ & $15.6 \pm 5.7$ & $12.5 \pm 5.5$ & $7.0 \pm 5.0$ & $3.0 \pm 4.7$ & 247 & 10.8 \\
$\mathrm{X}$ & $3.2 \pm 5.5$ & $4.7 \pm 6.0$ & $4.0 \pm 5.8$ & $8.1 \pm 7.2$ & $11.7 \pm 10.3$ & 117 & 5.1 \\
\hline
\end{tabular}

Table 4: Relative fraction of each taxonomic class (by number of objects) from each source region defined by Bottke et al. (2002) and Greenstreet et al. (2012), compared with the distribution of taxonomic types among 3 to $5 \mathrm{~km}$ inner Main Belt (IMB) asteroids (where Q-types are merged with S-types, see DeMeo and Carry 2013). 


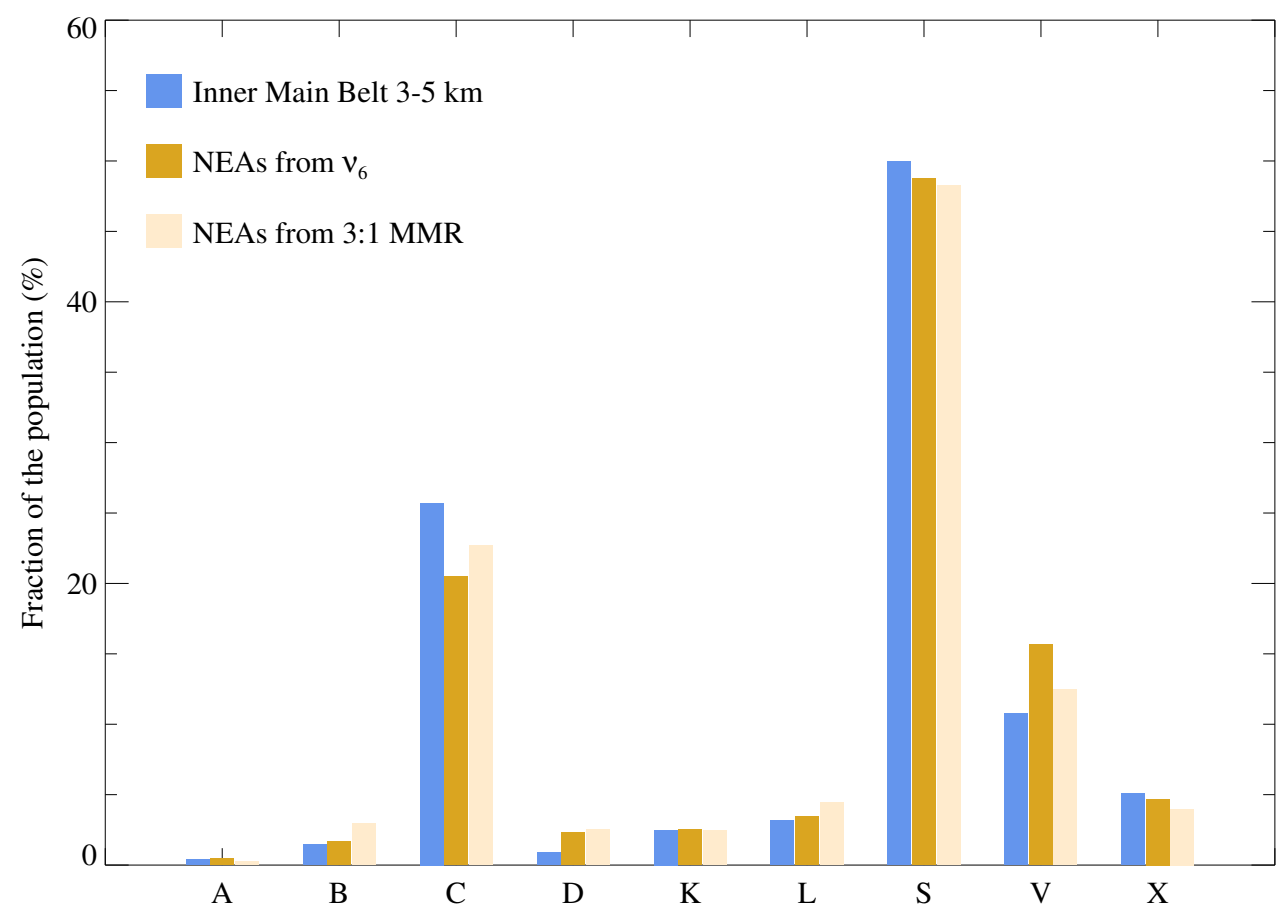

Figure 5: Relative fractions (by number) of taxonomic classes for asteroids in the inner part of the main-belt (2.0-2.5 au) with diameter between 3 and $5 \mathrm{~km}$ (computed from DeMeo and Carry 2013, 2014) compared with the predicted fractions originating from $\nu_{6}$ and 3:1 resonances (see Sec. 5) 


\section{Spectral slope and space weathering}

\subsection{Size dependence of spectral slope}

A size dependency of the spectral characteristics of S-type asteroids has been detected early on (e.g., Gaffey et al. 1993) and a correlation between size and spectral slope among NEAs and MCs was shown by Binzel et al. (2004) based on a sample of 187 S- and 20 Q-types. Independently of the other reddening effects due to grain size (see, e.g., Cloutis et al. 2015) and solar phase angle (Bell et al. 2002; Sanchez et al. 2012; Reddy et al. 2015), this trend has been associated with space weathering, causing the spectrum of Q-types, i.e., that of ordinary chondrites made of assemblage of olivine and pyroxenes, to redden into S-type spectra under the action of solar wind ions (see Sasaki et al. 2001; Strazzulla et al. 2005, among many others). Even if the timescale of this reddening is still debated (from $<10^{6} \mathrm{yrs}$ by, e.g., Vernazza et al. (2009) to more than $10^{9}$ yrs by, e.g., Willman and Jedicke (2011)), it is shorter than the age of the Solar System (see also Jedicke et al. 2004; Nesvorný et al. 2005; Willman et al. 2008, 2010; Dell'Oro et al. 2011; Marchi et al. 2010, 2012). Some regolith refreshing mechanisms must therefore be invoked to explain non- and less-weathered surfaces.

Because the collisional lifetime decreases with size, the surface is expected to be younger at smaller sizes. Smaller asteroids should therefore have a shallower spectral slope than larger bodies on average, albeit with a larger dispersion as collisions are a stochastic process (Binzel et al. 2004). This trend has been found recently for the small (diameter below $5 \mathrm{~km}$ ) members of the Koronis family in the main belt $(\approx 400$ objects, Rivkin et al. 2011; Thomas et al. 2011, 2012). We present in Fig. 6 the spectral slope of 467 
S-types plotted against their absolute magnitude. This sample is almost 3 times bigger than that of Binzel et al. (2004), and although we cannot directly compare the values of the spectral slope due to the different definitions and normalization wavelength, we note that we observe the same linear trend from 5 to $1 \mathrm{~km}$ (also visible in Thomas et al. 2012). However, we do not observe an increase of the standard deviation toward smaller diameters nor a "saturation" regime below $1 \mathrm{~km}$ as visible in their Fig. 7. If this trend seems to persist below $1 \mathrm{~km}$, the statistical relevance of this information decreases, however, as the number of objects drops dramatically, both here and in Binzel et al. (2004).

We interpret the difference between our findings and those reported by Binzel et al. (2004) as the effect of the larger sample, in which the signal to noise ratio of the data is roughly constant over the entire size range: contrarily to the spectral observations, generally noisier for small objects, the limiting factor of the present SDSS data is the apparent $z^{\prime}$ magnitude. Objects with four-bands photometry were brighter than magnitude 20 at the time of their observations, that is 2 magnitudes brighter than the limiting magnitude in $\mathrm{g}^{\prime}, \mathrm{r}^{\prime}$, and $\mathrm{i}^{\prime}$, over which the spectral slope is computed.

Perhaps not surprisingly, a similar trend is visible for the 38 Q-types displayed in Fig. 6. These represent the youngest surfaces of their size range. Following the argument above, larger asteroids are refreshed less often than smaller objects, and this also applies to Q-types on their path to redden into S-types, independently of the mechanism that originally reset their surface. 


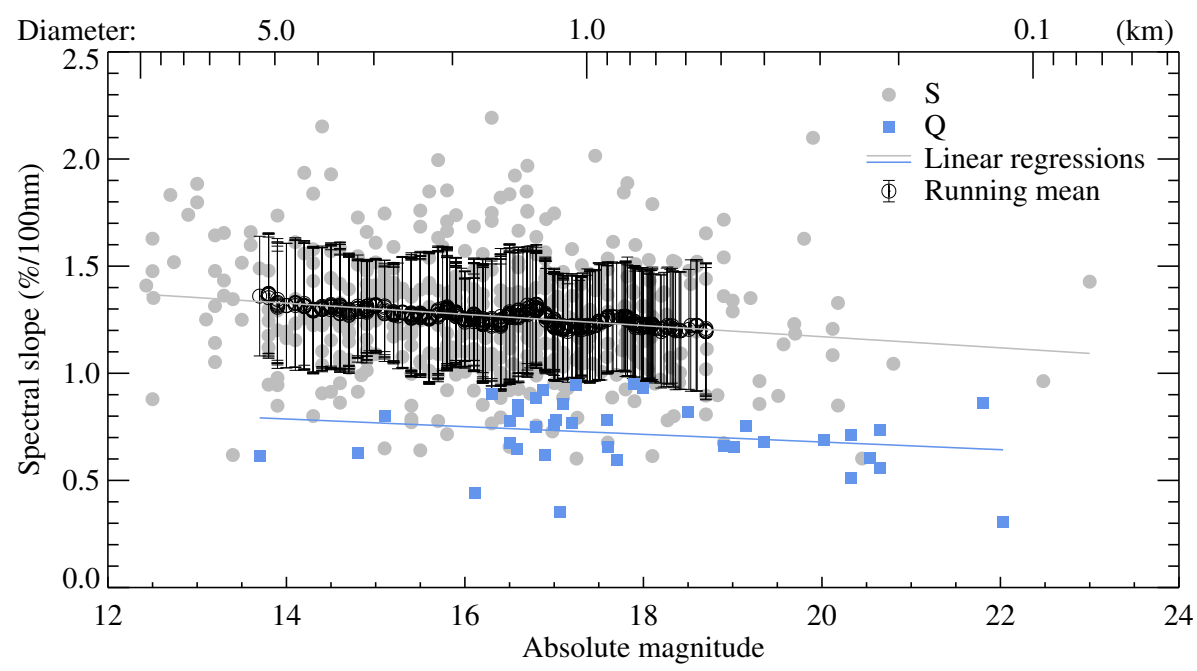

Figure 6: Spectral slope of $467 \mathrm{~S}-$ and 38 Q-types against their absolute magnitude (H). An approximative conversion to diameter is also reported (see Fig. 3). For both, a linear regression is presented, together with a running-window average (window size 50) for the sample of S-types.

\subsection{Planetary encounters refresh surfaces}

If collisions play a stochastic role in modulating the spectral slope of silicate-rich asteroids (S-, A-, V-, Q-types), the question on the main rejuvenating process is still open. Binzel et al. (2010) and DeMeo et al. (2014b) have recently provided observational support to the mechanism proposed by Nesvorný et al. (2005) of close encounters with terrestrial planets. The tidal stress during the close encounters has been proposed to reveal fresh material (responsible for the Q-type appearance) via landslides and regolith shaking.

Both Binzel et al. (2010) and DeMeo et al. (2014b) investigated the or- 


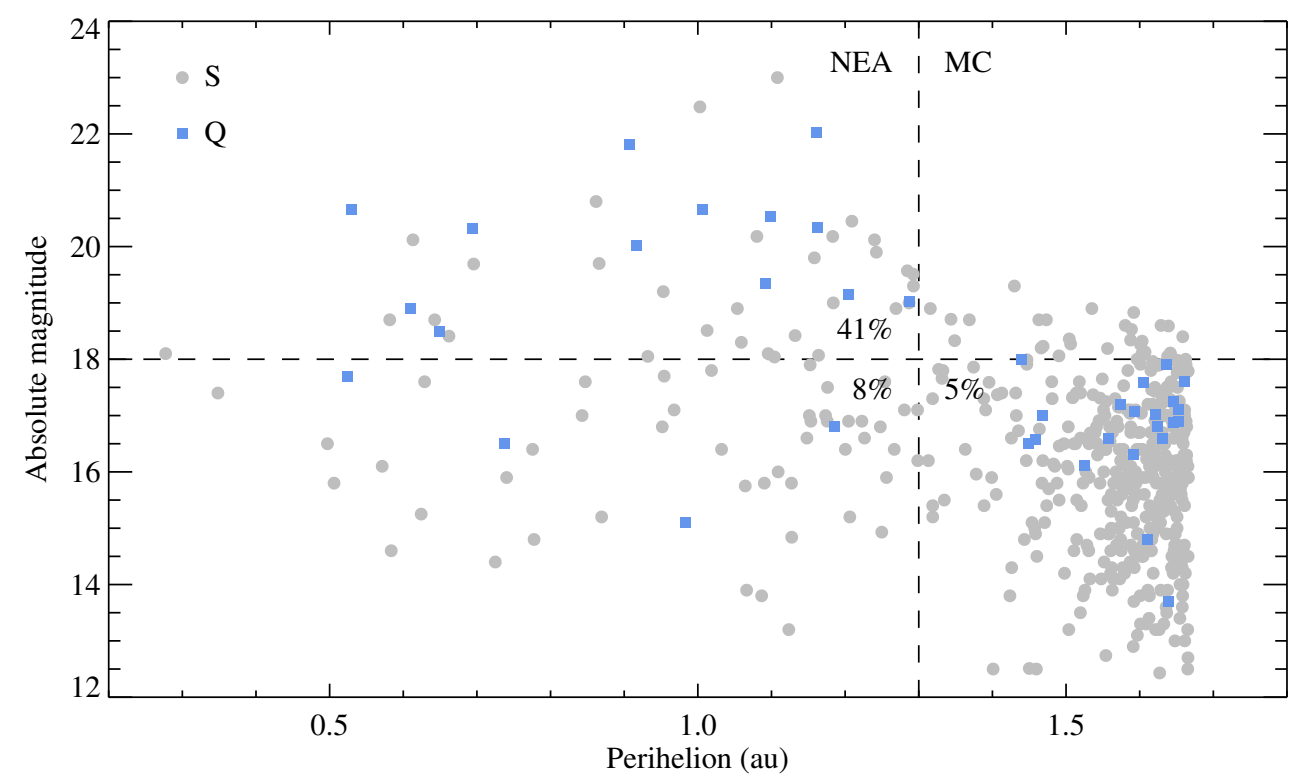

Figure 7: Absolute magnitude of S- and Q-types asteroids against perihelion distance. Below $\mathrm{H}=18$ (95\% of our MC sample), the Q/S fraction among NEAs and MCs is roughly similar (5-8\%). The Q/S fraction rapidly increase for smaller diameters $(\mathrm{H}>18)$ among NEAs, and many Q-types will be discovered in the sub-kilometer population of MCs.

bital history ${ }^{9}$ of two samples of near-Earth Q- and S-type asteroids, searching for planetary encounters "close enough" (up to a few lunar distances) to reset space weathering effect. As a results, all the Q-types they tracked had small MOID with either the Earth or Mars in the past 500,000 years, a time at which some level of space weathering should have already developed (see Sect. 6.1 above). A significant fraction of S-types had also small MOIDs with

\footnotetext{
${ }^{9}$ Trajectories of 6 clones per asteroid with a difference of $10^{-6}$ au/yr in initial velocity in each direction were integrated with SWIFT3 RMVS (Levison and Duncan 1994) over 500 kyr, with a time step of 3.65 days. Minimum Orbital Insertion Distance (MOID) were averaged over 50 years.
} 
terrestrial planets. However, the MOID measures the distance between two orbits, and not between two bodies, and a small MOID does not necessarily implies encounters. The authors concluded that planetary encounters, with Mars and the Earth, could explain the presence of Q-types among NEAs (while they are rare among main-belt asteroids). They derived a putative range $r^{\star}$ of 16 Earth radii at which the resurfacing could be felt by asteroids.

Independently, Nesvorný et al. (2010) used the sample by Binzel et al. (2010), using a different approach. By tracking ${ }^{10}$ test particles from NEA source regions (similar to Bottke et al. 2002, in a way) to NEA space and using a simple step-function model for space weathering, they explored the possible range of planetary distances and space weathering timescales that would result in the amount and orbital distribution of the $\mathrm{Q} / \mathrm{S}$ ratio. They concluded on a smaller sphere of influence of planets, with $r^{\star}$ between 5 and 10 Earth radii. Contrarily to Binzel et al. (2010), who only addressed Earth encounters, they found encounters with Venus were as effective as those with the Earth. They finally found that encounters with Mars were less important, and predicted a very small fraction of Q-types among Mars-crossers $(\lesssim 1 \%)$.

The 23 Q-types candidates we identified among Mars-crossers (Sec. 4) account for about $2.5 \%$ of the sample (and the Q/S ratio is about $5 \%$ ). This ratio is smaller than for NEAs where it reaches $20 \%$ (the total fraction of Q-types among NEAs is $8 \%$ ), but it is a lower limit. The Q/S fraction is indeed strongly diameter-dependent as illustrated in Fig. 7. When comparing

\footnotetext{
${ }^{10}$ Trajectories of 100 clones per asteroid, spread along the line of variation, were integrated also with SWIFT3 RMVS over 1 Myr, with a time step of 1 days.
} 
similar size range $(\mathrm{H}<18$, corresponding to $95 \%$ of the $\mathrm{MC}$ sample here), the Q/S fraction is roughly similar for NEAs and MCs, around 5-8\%. The ratio jumps to $40 \%$ for sub-kilometric NEAs, and many more Q-types could be discovered among sub-kilometric MCs. Because of this high fraction of Qtypes among MCs, challenging the prediction by Nesvorný et al. (2010), we first derive the theoretical radius of influence during an planetary encounter by studying the forces acting on surface grains (Sec. 6.2.1 to Sec. 6.2.4) and then study the dynamical history of all the S- and Q-types asteroids presented here, recording their close encounters with massive bodies (Sec. 6.2.5).

\subsubsection{Resurfacing model}

While resurfacing of asteroids by planetary encounters has already been studied (Nesvorný et al. 2005, 2010; Binzel et al. 2010; DeMeo et al. 2014b), the physics of the surface was not given much attention in the aforementioned articles. The velocity and duration of the encounter, the object's shape, internal structure, surface gravity, local slopes, rotation rate and orientation, and the nature of the pre-existing regolith and its cohesion, were listed as possible parameters dictating the distance at which an encounter can resurface the asteroid.

In the following we are interested in finding the mean processes responsible for resurfacing and the minimum close encounter distances at which it would occur. We thus consider a simple force balance equation describing the accelerations a surface particle is likely to experience at the moment of closest approach. A particle on the asteroid's surface is subject to the following 
forces during a flyby (e.g., Hartzell and Scheeres 2011) :

$$
F_{t d}+F_{c f}+F_{e s}+F_{l t}=F_{g a}+F_{c o}+F_{s p}
$$

On the left-hand side of equation (1) we have summed all the forces that can displace the particle: $F_{t d}$ are the tidal forces due to the planetary encounter, $F_{c f}$ is the centrifugal pseudo force due to the asteroid's rotation, $F_{e s}$ is a repulsive electrostatic force that originates from the electric charging of surface particles, and $F_{l t}$ is the displacement force acting when the asteroid's rotation state changes (librational transport, see Yu et al. 2014). The right-hand side contains forces that can keep a particle in place: $F_{g a}$ is the self-gravity of the asteroid, $F_{c o}$ is the cohesion between surface particles, and $F_{s p}$ is the solar radiation pressure. We describe the force model in detail in Appendix A and show in Fig. 8 their absolute magnitude as function of the diameter of surface grains.

To determine the minimum planet-to-asteroid distances that would result in the resurfacing of the asteroid, we consider two limiting cases (see Fig. 9). Easy and hard cases are defined based on whether the conditions for resurfacing are favorable or not, respectively. By doing so, we aim at deriving limits to distinguish regions in the parameter space where resurfacing is practically guaranteed, from regions that will leave the asteroid's surface untouched.

\subsubsection{Easy resurfacing conditions}

If the asteroid has a rotation rate close to the spin-barrier and it passes the planet with its spin axis perpendicular to the orbital plane of the hyperbolic encounter, i.e., with zero obliquity with respect to the planet, resurfacing is 


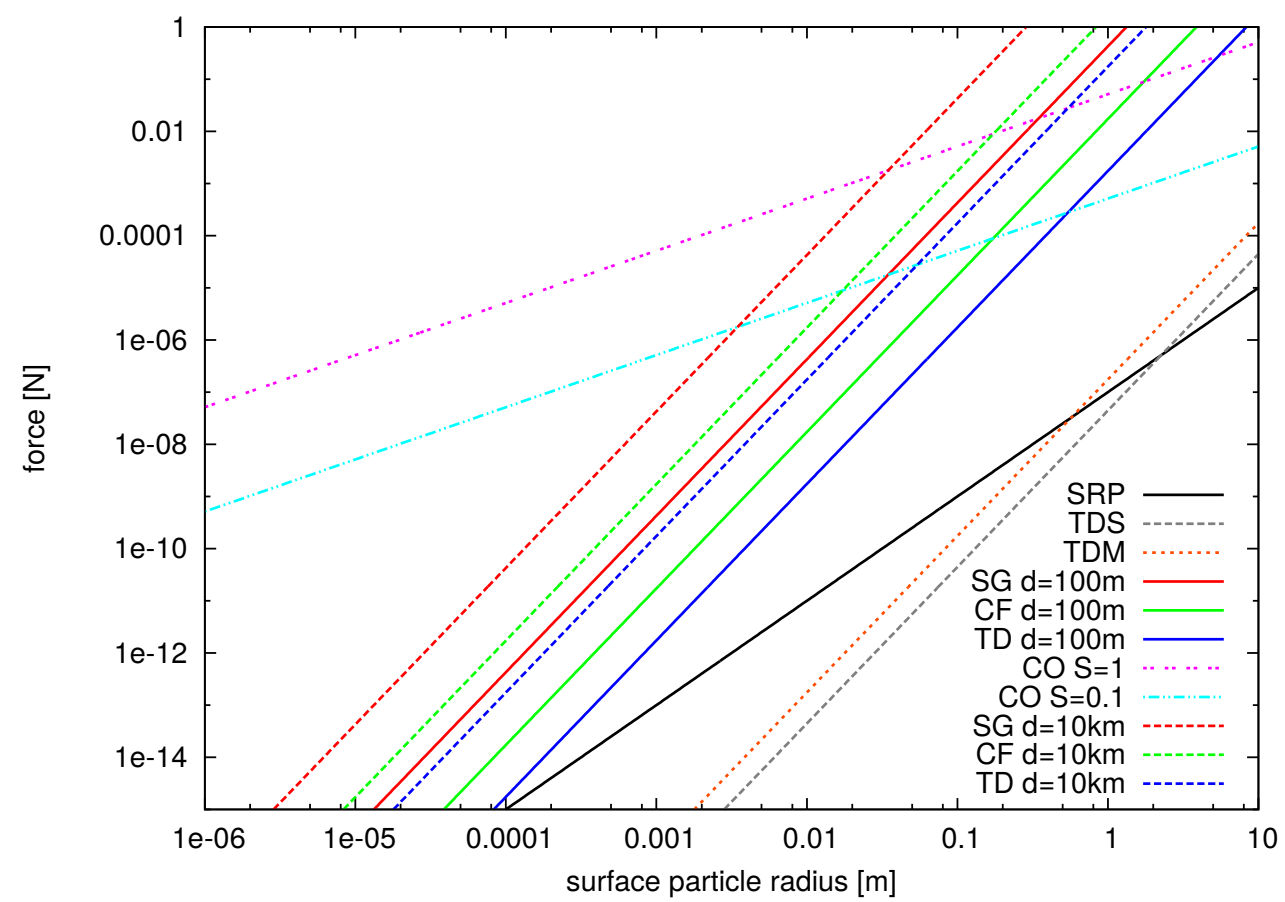

Figure 8: Comparison of the forces acting on surface particles as function of their diameter during an encounter with the Earth at a 10 Earth-radii distance. The different lines represent the absolute magnitude of the following forces: self gravity (SG), cohesion (CO), centrifugal forces $(\mathrm{CF})$, solar radiation pressure (SRP) for two different asteroid diameters, $100 \mathrm{~m}$ and $10 \mathrm{~km}$, with the same bulk density $\left(1900 \mathrm{~kg} \cdot \mathrm{m}^{-3}\right)$ and rotation period $(P=10 \mathrm{~h})$. Tidal accelerations due to the moon (TDM) and the sun (TDS) are only visible for the asteroid with a $100 \mathrm{~m}$ diameter. The range of likely cohesive forces is represented by the choice of cleanliness parameters between 0.1 and 1 (Perko et al. 2001; Scheeres et al. 2010; Hartzell and Scheeres 2011; Sánchez and Scheeres 2014). One can see that cohesion dominates all other forces for particle sizes below $10^{-3} \mathrm{~m}$.

more likely. Since fast rotators are stable with regard to perturbations of the spin state, we can assume that the initial spin vector remains constant and librational transport will not play a role. Low self-gravity is also conducive to 


\section{Easy conditions}

\section{To massive body}

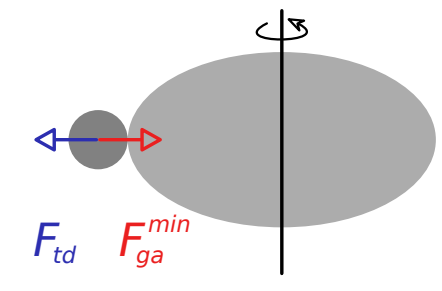

$F_{c f}$

\section{Hard conditions}

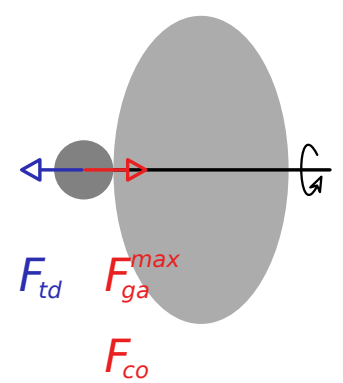

Figure 9: Schematic view of the easy (6.2.2) and hard (6.2.3) conditions for resurfacing. The forces acting on a surface particles are: $F_{t d}$ are the tidal forces due to the planetary encounter, $F_{c f}$ is the centrifugal force due to the asteroid's rotation, $F_{g a}$ is the self-gravity of the asteroid, and $F_{c o}$ is the cohesion between surface particles.

resurfacing. The effect of solar radiation pressure can be neglected, because it is orders of magnitude weaker than the other contributions.

Considering the high porosity of the first layers of asteroid surfaces Vernazza et al. (2012) that could originate from the electrostatic charging of the surface particles, we deem cohesion between surface particles to be negligible in this case. Since electrostatic inter-particle repulsion is already incorporated in the assumption of a highly porous upper layer of regolith, no additional electrostatic forces shall be considered. As a consequence of the above assumptions that yield the best case scenario in terms of resurfacing an asteroid, equation (1) simplifies to

$$
F_{t d}+F_{c f}^{\max }=F_{g a}^{\min }
$$

Inserting the forces discussed in Appendix A into equation (2), we find the distance $r^{\star}$ between the asteroid and the planet where all accelerations cancel. In other words, $r^{\star}$ is the largest planet-to-asteroid distance at which 
a particle is no longer bound to the asteroid. If we assume the asteroid to be a tri-axial ellipsoid defined by $R_{a} \geq R_{b} \geq R_{c}$, rearranging its surface can take place at a planetary distance of

$$
r_{\text {easy }}^{\star}=\left(\frac{6 \mathcal{G} M}{4 \pi \mathcal{G} \beta \rho-3 \omega_{s b}^{2}}\right)^{1 / 3}
$$

where $\beta=R_{b} R_{c} / R_{a}^{2}$ is a shape factor relating the three principle axes. Equation (3) describes a particle that is on the point of the surface farthest away from the center of rotation. It is easy to see that for any sort of ellipsoid shape the denominator in equation (3) shrinks. Therefore, ellipsoid shapes have extended resurfacing distances compared to spherical shapes (in which $\beta=1)$. Also, $r_{\text {easy }}^{\star}$ can become arbitrarily large when $\omega=\omega_{s b}=\sqrt{4 \pi G \beta \rho / 3}$ (with $\rho$ the asteroid bulk density), i.e., when the asteroid's spin reaches the spin barrier (e.g., Pravec et al. 2006). Since an asteroid is expected to have shed most of its surface material at the spin barrier (Holsapple 2007), resurfacing will become impossible. Therefore, we will only consider rotation states slightly below this limit $(2 \pi / \omega=P \sim 2.5 \mathrm{~h})$.

\subsubsection{Hard resurfacing conditions}

Resurfacing becomes most difficult, on the other hand, if the asteroid has basically no rotation or an obliquity close to $90^{\circ}$ during its flyby. Then, there is no centrifugal acceleration that facilitates the collapse of rubble pile columns or lifts particles off the surface. Resurfacing is also more difficult if the asteroid mass is high, enhancing its self gravity. If electic charges of surface aggregates are feeble, the particles may settle and interlock in dense configurations that are dominated by cohesion rather than by electrostatic repulsion (Scheeres et al. 2010). Finally, for non-rotating bodies a change in 
the asteroid rotation is likely to occur due to the dynamical instability of a non-rotating configuration during flybys. Hence, librational transport could, in principle, occur. Yet, since we are interested in the case where resurfacing is most difficult, we will neglect its contribution regardless. The equation describing a scenario when resurfacing is most difficult thus writes:

$$
F_{t d}=F_{g a}^{\max }+F_{c o}
$$

Following the same approach as for easy resurfacing and using equation (4) to derive $r_{\text {hard }}^{\star}$ leads to planetary distances that are far below the asteroid's disruption regime. Indeed, cohesive forces are dominating all other contributions for particle sizes below $10^{-3} \mathrm{~m}$, as visible in Fig. 8, since $99 \%$ of the particles that cover the surface have radii below $10^{-4} \mathrm{~m}$ (Appendix A.4). Consequently equation (4) is not a good estimator for the resurfacing limit. Since resurfacing is guaranteed when the asteroid enters the deformation or even disruption regime, we can simply use asteroid's Roche limit as a proxy for the conservative resurfacing distance. If we assume a perfectly spherical asteroid without rotation we have the following force equilibrium

$$
F_{g a}=F_{t d}
$$

and consequently

$$
r_{\text {hard }}^{\star}=R\left(\frac{2 M}{m}\right)^{1 / 3} \approx\left(\frac{3 M}{2 \pi \rho}\right)^{1 / 3},
$$

where once again, $R$ is the asteroid's (maximum) radius, and $m$ and $M$ are the asteroid and the planet masses. The simple spherical Roche limit serves as a proxy for $r_{\text {hard }}^{\star}$, as it would hold as a lower boundary should the asteroid be an ellipsoid. 


\subsubsection{Planet to NEA distance for resurfacing}

We use the two relations determined above for easy and hard resurfacing to evaluate the minimum distance at which a planetary encounter can displace surface particles. We compute the easy and hard cases for the following parameters: an absolute magnitude of 20 (corresponding to diameter of 200 and $600 \mathrm{~m}$ for albedo of 0.5 and 0.05 respectively), a bulk density of 1.9 and $2.7 \mathrm{~g} . \mathrm{cm}^{-3}$, and a shape factor $\beta$ of 0.21 . We show these threshold distances for Venus, Earth, and Mars, as function of the asteroid rotation period in Fig. 10.

First, the difference between the easy and hard resurfacing distances is substantial, especially for fast rotator. Then, we find distances ranging from a couple of planetary radii up to 10 planetary radii in extreme cases, when the rotation period is close to the spin barrier. This is fully compatible, yet slightly lower, than the estimates of 5-10 planetary radii from Nesvorný et al. (2010) derived independently. This implies that resurfacing through planetary encounters is not common, as the encounters have to be very close.

However, one might ask whether it is permissible to simply ignore the effect of cohesion, as has been done in equation 5. In fact, Figure 8 shows that cohesion dominates other forces up to millimeter sized particles. However, for larger particles this is no longer the case. If the asteroid comes close enough during its approach, tidal forces will then be strong enough to lift decimeter- to meter-sized objects on the surface of the asteroid. While objects of this size do not contribute significantly to the overall surface of the asteroid (Sánchez and Scheeres 2014), they can refresh the surface if displaced by triggering landslides for instance. 
A similar line of thought can be used to argue that we may be able to ignore cohesive and tensile effects in the case of non-spherical objects, since the internal stresses decay rapidly towards the surface (Holsapple 2007; Sánchez and Scheeres 2014). Such an approach would not be permissible, if we were searching for criteria to describe global deformation or complete asteroid failure. There, one would have to account for internal cohesion and material stresses (Sharma et al. 2006). Yet, as we are merely interested in whether the combination of forces acting during a close encounter can displace any sort of particle on the surface of the asteroid, we argue that our simplified approach is valid.

\subsubsection{Dynamical simulations}

We investigate the hypothesis of resurfacing by planetary encounters developed above by testing whether there is a significant difference in the number of potential resurfacing events between Q- and S-type asteroid samples. We probe the dynamical history of each asteroid by propagating its position together with a sample of 96 clones 0.5 Myrs into the past using a symplectic integrator based on Yoshida's T+V split (Yoshida 1990) with General Relativity (GR) correction (Lubich et al. 2010). Symplectic integrators have the advantage that the error in the mean anomaly, i.e., the position on the planet on its orbit, does not grow as quickly as with standard propagators (Eggl and Dvorak 2010, and references therein). Using an $8^{\text {th }}$ order integrator with a stepsize of 1 day in the drift in mean anomaly over 0.5 Myrs is less than $0.015^{\circ}$ in the two-body problem Sun-Earth, corresponding to a total along track displacement of less than 6 Earth radii. As for close encounters, the propagator is able to resolve all close encounters lasting more than 6 


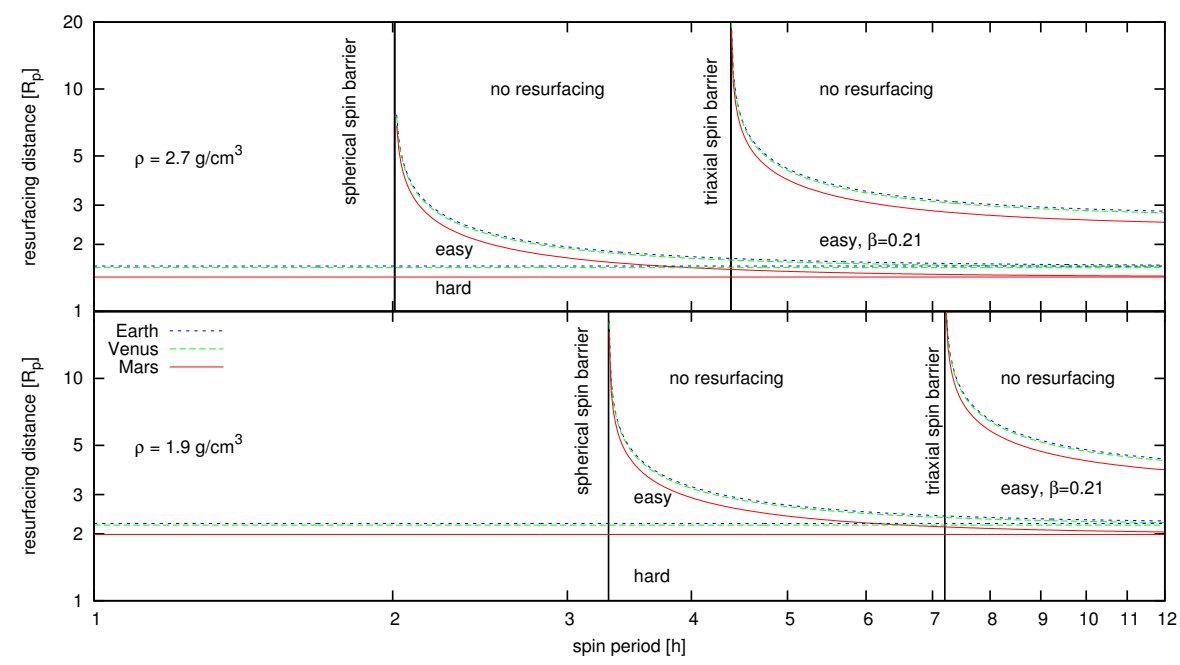

Figure 10: Best case $\left(r_{\text {easy }}^{\star}\right)$ and worst case $\left(r_{\text {hard }}^{\star}\right)$ resurfacing distances in planetary radii for an asteroid (see text for details) that has close encounters with Mars, Venus and the Earth. Resurfacing is possible for close encounter distances below the respective lines. Note that forces scale with asteroid diameter and this picture is therefore valid for any NEA size.

hours reasonably well. The limit resolution is reached for encounters lasting three hours. While the integration algorithm is not regularized, care has been taken to avoid losing accuracy due to imprecise calculation of accelerations and the accumulation of round off errors (e.g., via the use of Kahan summation).

Each simulation contained the following massive perturbers: the 8 planets, the barycenter of the Pluto system as well the major asteroids (1) Ceres, (2) Pallas, (4) Vesta and (10) Hygiea, henceforth referred to as the massive bodies. Initial conditions for the massive bodies were taken from JPL 
DE405 ephemerides at the epoch J2000. The initial conditions for the asteroids were constructed using the open source software OrbFit ${ }^{11}$ (Milani et al. 2008), taking all observations up to April 2014 into account. Asteroid orbits were fit to the non-relativistic dynamical system containing all planets and the Pluto system. No asteroid perturbers were taken into account during the fitting process. Given the timescales involved in the orbit fitting and differential correction, the discrepancies arising from neglecting GR and the major perturbing asteroids on the investigated asteroids can be considered small compared to the orbit uncertainties.

The uncertainty covariance matrix resulting from the orbital fit was then sampled along the line of variation (Milani et al. 2000) from $-3 \sigma$ to $+3 \sigma$ with 96 clones per asteroid. The 96 clones were then propagated together with the nominal orbit in order to see the dispersion in phase space. All close encounters, with the Earth, Venus, Mars, Jupiter and the main perturbing asteroids were cataloged for each clone in form of close minimum encounter distance (MED) and time histograms. The following limit distances were used to trigger a close encounter log: Jupiter: $2.56956 \mathrm{au}$, Venus, Earth and Mars: $0.256956 \mathrm{au}$, the main perturbing asteroids: $0.0256956 \mathrm{au}$. These values correspond to approximately 7 Hill's radii for Jupiter, 25 for the Earth and Venus, 35 Hill's radii for Mars and 35-70 Hill's radii for the perturbing asteroids. Minimum encounter distances and velocities were calculated using cubic spline interpolation of the asteroid's orbit during its close encounter. Global minimum and average encounter distances together with their vari-

\footnotetext{
11 http://adams.dm.unipi.it/orbfit/
} 
ances were also saved. In addition, minimum orbit intersection distances (MOIDs) were calculated every 800 days. Similarly to the MED values, MOID histograms, averages and variances were cataloged.

It should be mentioned that results stemming from integrating NEO orbits backwards in time need to be interpreted with care. Similarly to forward propagation NEAs on chaotic trajectories with the terrestrial planets have a relatively short horizon that limits an accurate prediction of their dynamical evolution (see, e.g., Michel 1997) In such cases even robust ensemble statistics will not yield reliable estimates on close encounter distances since the divergence of nearby solution becomes exponential. As a consequence, we decided to exclude those NEAs from the statistics as soon as the spread in clone encounter distances becomes large. In our dynamical study, we also excluded the Yarkovsky effect for the following reasons. First, the spin state and orientation of the chosen targets are largely unknown. While this could be remedied using a statistical distribution of drift parameters among the clones of each NEA as proposed, for instance, by Spoto et al. (2014), this would only lead to an artificially increased spread in 96 clones making it harder to determine which NEAs are on chaotic orbits and which are not. Second, close encounters can change the spin state, making self-consistent predictions very difficult. That being said, Yarkovsky drift rates of our targets range between $10^{-4}$ and $10^{-3}$ au/Myr. The cross-track error that results from neglecting the Yarkovsky drift alone can range between 2-20 Earth radii over 0.5 Myrs, and along track position errors are much larger.

Regardless of the simplifications of our dynamical model, we find that all the Q-types have a minimum MOID allowing a close encounter with the 
Earth (18\% of the sample), or Mars (100\%) in the past 500,000 years (we use the median values from all the clones here). A large fraction of the Stype sample also present a minimum MOID that could have led to a close encounter with one of the terrestrial planets $(12.9 \%, 6.7 \%$, and $95.4 \%$ for the Earth, Venus, and Mars respectively), similarly to the situation presented by (Binzel et al. 2010) and (DeMeo et al. 2014b). However, a small MOID does not necessary imply a close encounter: the MOID provides a measure of the distance between the orbits, not between the bodies. The typical example would be a Trojan asteroid, for which the MOID is small by definition, but that would never encounter the planet.

To overcome this issue, we also study the minimum encounter distance (MED) of the asteroids and their clones: that is the real distance between the particles and the massive bodies. However, even with a symplectic integrator, the drift in mean anomaly steadily increases with ephemeris time, and reach the level of a few planetary radii, at which the resurfacing is deemed to occur (see 6.2.4 above). Results for MED therefore potentially suffer from an underestimation of the number of close encounters, especially for the closest. The total number of detected MED resurfacing events is much less than the theoretically favorable number of configurations given by the encounter numbers using MOIDs. However, MEDs share the same trends with our MOID results.

The analysis using the median properties of each asteroid with its 96 clones seems therefore not conclusive: based on MED values, the planetary encounters are not expected to play any role in rejuvenating the surface of the Q-types, and based on MOID values, there should be more fresh sur- 
faces (i.e., less S-types) as the vast majority of our sample present small MOID with Mars. We therefore study the populations of S- and Q-types statistically. We present in Fig. 11 the cumulative number of close encounters reaching the planetary distance for easy and hard resurfacing scenarios (see 6.2.2 and 6.2.3) between our sample of asteroids and Venus, the Earth, and Mars. There is a clear difference in the number of encounters experienced by the population of Q-types compared to the S-types sample for both Venus and the Earth. That is, the asteroids presenting a fresh surface, similar to ordinary chondrites and exempt of signature from space weathering, tend to have more close encounters with massive bodies that those asteroids with space-weathered surface. Significant resurfacing would thus occur from the stress during repetitive close encounters with planets. The distribution of encounters for both the Earth and Venus clearly highlights the sample of Q-types among the NEAs sample presented here (and somewhat provides an a posteriori validation of our classification into S- and Q-types, the two groups being apparently dynamically different).

Conversely, the encounter distribution with Mars is similar for both taxonomic classes, as both could theoretically have numerous encounters with Mars, within the expected distance suitable for resurfacing to occur. Among all the parameters recorded during the dynamical simulation, none showed a significant difference between the two populations and we are therefore limited to speculations. Because the sample of MCs studied here exhibit larger diameters than the NEAs encountering the Earth, they are deemed to be older. Their surface may therefore have reached the saturation state introduced by Marchi et al. (2012), where rejunevation is inefficient. The frequent 


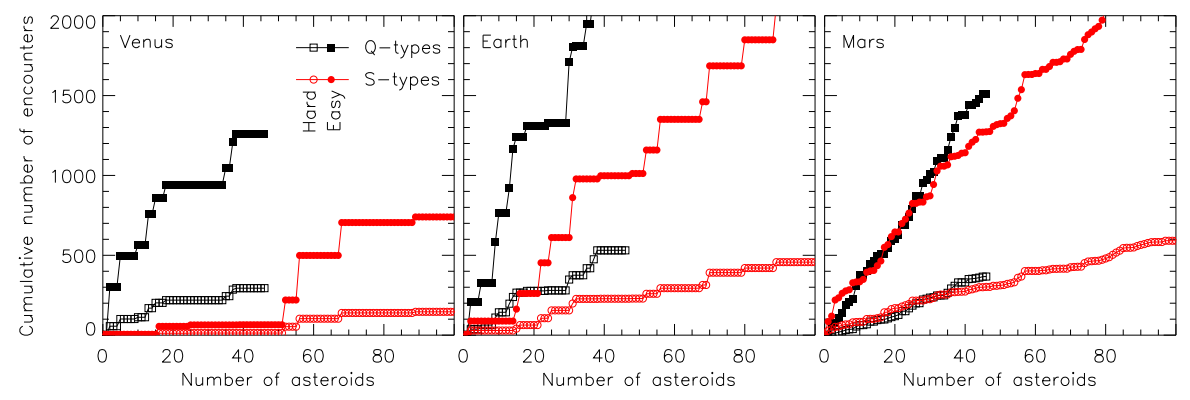

Figure 11: Comparison of the number of close encounters for the easy and hard resurfacing cases (filled and open symbols) for the samples of Q- and S-type asteroids (in black squares and red circles).

encounters with Mars would then no longer turn the S-type surfaces into Qtypes. Another possibility is that the limit for easy resurfacing being tight to the rotation period and obliquity during the close encounter, only a small fraction of close encounters do trigger resurfacing events, with a preference for fast-spinning asteroids. A survey of rotation period of Q-types among NEAs and MCs population could address this point.

\section{Conclusion}

In this work, we report on the dynamical and surface properties of nearEarth asteroids (NEAs) and Mars-crosser asteroids (MCs), based on the analysis of their colors in the visible. Our sample includes:

- 43 NEAs and 310 MCs listed in the Moving Object Catalogue (MOC4) of the Sloan Digital Sky Survey (SDSS);

- 206 NEAs and 776 MCs, in publicly available images of the SDSS, using our citizen-science project "Near-Earth Asteroids Recovery Program" 
of the Spanish Virtual Observatory (SVO, Solano et al. 2013) measured in four filters $\left(\mathrm{g}^{\prime}, \mathrm{r}^{\prime}, \mathrm{i}^{\prime}\right.$, and $\left.\mathrm{z}^{\prime}\right)$;

- 678 NEAs and MCs asteroids measured in three filters $\left(\mathrm{g}^{\prime}, \mathrm{r}^{\prime}\right.$, and $\left.\mathrm{i}^{\prime}\right)$ also from our citizen-science project, for 254 of which we assign tentative taxonomic classification.

In total we have determined the taxonomic class of these 982 NEAs and MCs using the DeMeo-Carry taxonomy for SDSS colors (DeMeo and Carry 2013), that is compatible with the Bus-DeMeo taxonomy based on V+NIR spectra (DeMeo et al. 2009)

The sample of taxonomic classes presented here correspond to an increase of known classes by $40 \%$ and $600 \%$ for NEA and MC populations, respectively. Among those, 36 NEAs can be considered potential targets for space missions, owing to their low $\delta v$. Some candidates for rare taxonomic classes such as D-, L-, and K-types are present within this sample and would benefit from further spectral investigations.

We then use the sample of asteroids with taxonomic classes based on fourfilter observations to study their source regions and the effect of planetary encounters on their surface properties. To this end

○ we compare the distribution of taxonomic classes between our sample of NEAs and MCs and the source regions, using the predictions resulting from the dynamical model presented by Greenstreet et al. (2012). The population of $2-5 \mathrm{~km}$ diameter asteroids in the main belt (DeMeo and Carry 2014) match closely the predictions from the sample presented here, supporting that the $\nu_{6}$ secular resonance and the 
3:1 mean-motion resonance with Jupiter are the primary sources of kilometer-size NEAs;

○ we analyze the dependence of spectral slope on diameter for asteroids in the S-complex. A linear trend of shallower slope toward higher absolute magnitude is found;

○ we develop a simple force model on surface grains during a planetary encounter;

○ we investigate the planetary distance at which a resurfacing event is expected to occur, by considering two extreme cases to the simple force model, when the conditions are the most or conversely the least conducive to favorable resurfacing event. This distance is found to be a function of the rotation period and density of the asteroid, and of the spin obliquity during the encounters. It ranges from 2 to $\approx 10$ planetary radii for Venus, the Earth, and Mars. Such values are consistent with previous estimates by Binzel et al. (2010) and Nesvorný et al. (2010);

○ we study the dynamical history of the sample of S- and Q-type by propagating their positions backward in time for 0.5 Myrs, together with 96 clones, using a symplectic post-Newtonian integrator. The population of Q-type presents statistically more encounters with Venus and the Earth at distance where resurfacing should occur than S-types. However, both populations present a high number of encounters with Mars and are indistinguishable. 


\section{Acknowledgments}

We thanks S. Greenstreet for providing the source region mapper extended to include Mars-crosser space. We acknowledge support from the Faculty of the European Space Astronomy Centre (ESAC) for B. Carry's visit. S. Eggl would like to acknowledge the support of the European Commission H2020-PROTEC-2014 grant no. 640351 (NEOShield-2). This publication makes use of the NEAs Precovery Service, developed under the Spanish Virtual Observatory project (Centro de Astrobiologia, INTA-CSIC) supported from the Spanish MICINN through grants AyA2008-02156 and AyA201124052. This work was granted access to the HPC resources of MesoPSL financed by the Region Ile de France and the project Equip@Meso (reference ANR-10-EQPX-29-01) of the programme Investissements dAvenir supervised by the Agence Nationale pour la Recherche. This material is based upon work supported, in part, by the National Science Foundation under Grant 0907766. Any opinions, findings, and conclusions or recommendations expressed in this material are those of the authors and do not necessarily reflect the views of the National Science Foundation.

This publication makes use of data products from the Wide-field Infrared Survey Explorer, which is a joint project of the University of California, Los Angeles, and the Jet Propulsion Laboratory/California Institute of Technology, funded by the National Aeronautics and Space Administration. Funding for the creation and distribution of the SDSS Archive has been provided by the Alfred P. Sloan Foundation, the Participating Institutions, the National Aeronautics and Space Administration, the National Science Foundation, the U.S. Department of Energy, the Japanese Monbukagakusho, and the Max 
Planck Society. The SDSS Web site is http://www.sdss.org/.

\section{References}

Abell, P., Barbee, B., Mink, R., Adamo, D., Alberding, C., Mazanek, D., Johnson, L., Yeomans, D., Chodas, P., Chamberlin, A., et al., 2012. The near-earth object human space flight accessible targets study (nhats) list of near-earth asteroids: identifying potential targets for future exploration. In: AAS/Division for Planetary Sciences Meeting Abstracts. Vol. 44.

Abell, P., Mazanek, D., Reeves, D., Naasz, B., Cichy, B., Nov. 2015. NASA's Asteroid Redirect Mission (ARM). In: AAS/Division for Planetary Sciences Meeting Abstracts. Vol. 47 of AAS/Division for Planetary Sciences Meeting Abstracts. p. 312.06.

Barucci, M. A., Cheng, A. F., Michel, P., Benner, L. A. M., Binzel, R. P., Bland, P. A., Böhnhardt, H., Brucato, J. R., Campo Bagatin, A., Cerroni, P., Dotto, E., Fitzsimmons, A., Franchi, I. A., Green, S. F., Lara, L.-M., Licandro, J., Marty, B., Muinonen, K., Nathues, A., Oberst, J., Rivkin, A. S., Robert, F., Saladino, R., Trigo-Rodriguez, J. M., Ulamec, S., Zolensky, M., Apr 2012. MarcoPolo-R near earth asteroid sample return mission. Experimental Astronomy 33, 645-684.

Bell, J. F., Izenberg, N. I., Lucey, P. G., Clark, B. E., Peterson, C., Gaffey, M. J., Joseph, J., Carcich, B., Harch, A., Bell, M. E., Warren, J., Martin, P. D., McFadden, L. A., Wellnitz, D., Murchie, S., Winter, M., Veverka, J., Thomas, P., Robinson, M. S., Malin, M., Cheng, A., Jan. 2002. Near- 
IR Reflectance Spectroscopy of 433 Eros from the NIS Instrument on the NEAR Mission. I. Low Phase Angle Observations. Icarus 155, 119-144.

Berthier, J., Vachier, F., Thuillot, W., Fernique, P., Ochsenbein, F., Genova, F., Lainey, V., Arlot, J., jul 2006. SkyBoT, a new VO service to identify Solar System objects. In: C. Gabriel, C. Arviset, D. Ponz, \& S. Enrique (Ed.), Astronomical Data Analysis Software and Systems XV. Vol. 351 of Astronomical Society of the Pacific Conference Series. p. 367.

Binzel, R., Reddy, V., , Dunn, T., 2015. The Near-Earth Object Population: Connections to Comets, Main-Belt Asteroids, and Meteorites. Asteroids IV.

Binzel, R. P., Apr. 2000. The Torino Impact Hazard Scale. Planetary and Space Science 48, 297-303.

Binzel, R. P., Morbidelli, A., Merouane, S., DeMeo, F. E., Birlan, M., Vernazza, P., Thomas, C. A., Rivkin, A. S., Bus, S. J., Tokunaga, A. T., Jan. 2010. Earth encounters as the origin of fresh surfaces on near-Earth asteroids. Nature 463, 331-334.

Binzel, R. P., Rivkin, A. S., Stuart, J. S., Harris, A. W., Bus, S. J., Burbine, T. H., Aug. 2004. Observed spectral properties of near-Earth objects: results for population distribution, source regions, and space weathering processes. Icarus 170, 259-294.

Binzel, R. P., Xu, S., Apr. 1993. Chips off of Asteroid 4 Vesta: Evidence for the parent body of basaltic achondrite meteorites. Science 260 (5105), $186-191$. 
Bottke, W. F., Morbidelli, A., Jedicke, R., Petit, J.-M., Levison, H. F., Michel, P., Metcalfe, T. S., Apr 2002. Debiased Orbital and Absolute Magnitude Distribution of the Near-Earth Objects. Icarus 156, 399-433.

Bus, S. J., Binzel, R. P., July 2002. Phase II of the Small Main-Belt Asteroid Spectroscopic Survey: A Feature-Based Taxonomy. Icarus 158, 146-177.

Carry, B., Dec. 2012. Density of asteroids. Planetary and Space Science 73, 98-118.

Carvano, J. M., Hasselmann, H., Lazzaro, D., Mothé-Diniz, T., 2010. SDSSbased taxonomic classification and orbital distribution of main belt asteroids. Astronomy and Astrophysics 510, A43.

Chapman, C. R., Morrison, D., Zellner, B. H., may 1975. Surface properties of asteroids - A synthesis of polarimetry, radiometry, and spectrophotometry. Icarus 25, 104-130.

Cloutis, E. A., Sanchez, J. A., Reddy, V., Gaffey, M. J., Binzel, R. P., Burbine, T. H., Hardersen, P. S., Hiroi, T., Lucey, P. G., Sunshine, J. M., Tait, K. T., May 2015. Olivine-metal mixtures: Spectral reflectance properties and application to asteroid reflectance spectra. Icarus 252, 39-82.

Dandy, C. L., Fitzsimmons, A., Collander-Brown, S. J., Jun. 2003. Optical colors of 56 near-Earth objects: trends with size and orbit. Icarus 163, 363-373.

de León, J., Licandro, J., Duffard, R., Serra-Ricart, M., 2006. Spectral analysis and mineralogical characterization of 11 olivine pyroxene rich NEAs. Advances in Space Research 37, 178-183. 
de León, J., Licandro, J., Serra-Ricart, M., Pinilla-Alonso, N., Campins, H., Jul. 2010. Observations, compositional, and physical characterization of near-Earth and Mars-crosser asteroids from a spectroscopic survey. Astronomy and Astrophysics 517, A23.

Dell'Oro, A., Marchi, S., Paolicchi, P., Sep. 2011. Collisional evolution of near-Earth asteroids and refreshing of the space-weathering effects. Monthly Notices of the Royal Astronomical Society 416, L26-L30.

DeMeo, F., Carry, B., Jul 2013. The taxonomic distribution of asteroids from multi-filter all-sky photometric surveys. Icarus 226, 723-741.

DeMeo, F. E., Binzel, R. P., Carry, B., Polishook, D., Moskovitz, N. A., Feb. 2014a. Unexpected D-type interlopers in the inner main belt. Icarus 229, 392-399.

DeMeo, F. E., Binzel, R. P., Lockhart, M., Jan. 2014b. Mars encounters cause fresh surfaces on some near-Earth asteroids. Icarus 227, 112-122.

DeMeo, F. E., Binzel, R. P., Slivan, S. M., Bus, S. J., jul 2009. An extension of the Bus asteroid taxonomy into the near-infrared. Icarus 202, 160-180.

DeMeo, F. E., Carry, B., Jan. 2014. Solar System evolution from compositional mapping of the asteroid belt. Nature 505, 629-634.

Eggl, S., Dvorak, R., 2010. An introduction to common numerical integration codes used in dynamical astronomy. In: Dynamics of small solar system bodies and exoplanets. Springer, pp. 431-480. 
Emery, J. P., Brown, R. H., Jul. 2003. Constraints on the surface composition of Trojan asteroids from near-infrared $(0.8-4.0 \mu \mathrm{m})$ spectroscopy. Icarus 164, 104-121.

Emery, J. P., Brown, R. H., Jul. 2004. The surface composition of Trojan asteroids: constraints set by scattering theory. Icarus 170, 131-152.

Emery, J. P., Burr, D. M., Cruikshank, D. P., Jan. 2011. Near-infrared Spectroscopy of Trojan Asteroids: Evidence for Two Compositional Groups. Astronomical Journal 141, 25.

Fornasier, S., Dotto, E., Hainaut, O., Marzari, F., Boehnhardt, H., de Luise, F., Barucci, M. A., Oct. 2007. Visible spectroscopic and photometric survey of Jupiter Trojans: Final results on dynamical families. Icarus 190, $622-642$.

Fornasier, S., Dotto, E., Marzari, F., Barucci, M. A., Boehnhardt, H., Hainaut, O., de Bergh, C., Nov. 2004. Visible spectroscopic and photometric survey of L5 Trojans: investigation of dynamical families. Icarus 172, 221232 .

Fujiwara, A., Kawaguchi, J., Yeomans, D. K., Abe, M., Mukai, T., Okada, T., Saito, J., Yano, H., Yoshikawa, M., Scheeres, D. J., Barnouin-Jha, O. S., Cheng, A. F., Demura, H., Gaskell, G. W., Hirata, N., Ikeda, H., Kominato, T., Miyamoto, H., Nakamura, R., Sasaki, S., Uesugi, K., 2006. The Rubble-Pile Asteroid Itokawa as Observed by Hayabusa. Science 312, $1330-1334$. 
Gaffey, M. J., Burbine, T. H., Piatek, J. L., Reed, K. L., Chaky, D. A., Bell, J. F., Brown, R. H., Dec. 1993. Mineralogical variations within the S-type asteroid class. Icarus 106, 573-602.

Gil-Hutton, R., Brunini, A., Feb. 2008. Surface composition of Hilda asteroids from the analysis of the Sloan Digital Sky Survey colors. Icarus 193, 567571.

Gounelle, M., Spurný, P., Bland, P. A., Jan. 2006. The orbit and atmospheric trajectory of the Orgueil meteorite from historical records. Meteoritics and Planetary Science 41, 135-150.

Greenstreet, S., Ngo, H., Gladman, B., Jan. 2012. The orbital distribution of Near-Earth Objects inside Earth's orbit. Icarus 217, 355-366.

Hartzell, C. M., Scheeres, D. J., Nov. 2011. The role of cohesive forces in particle launching on the Moon and asteroids. Planetary and Space Science $59,1758-1768$.

Holmberg, J., Flynn, C., Portinari, L., Apr. 2006. The colours of the Sun. MNRAS 367, 449-453.

Holsapple, K. A., 2007. Spin limits of solar system bodies: From the small fast-rotators to 2003 el61. Icarus 187 (2), 500-509.

Holsapple, K. A., Apr. 2007. Spin limits of Solar System bodies: From the small fast-rotators to 2003 EL61. Icarus 187, 500-509.

Ivezić, Ž., Lupton, R. H., Jurić, M., Tabachnik, S., Quinn, T., Gunn, J. E., 
Knapp, G. R., Rockosi, C. M., Brinkmann, J., Nov. 2002. Color Confirmation of Asteroid Families. Astronomical Journal 124, 2943-2948.

Ivezić, Ž., Tabachnik, S., Rafikov, R., Lupton, R. H., Quinn, T., Hammergren, M., Eyer, L., Chu, J., Armstrong, J. C., Fan, X., Finlator, K., Geballe, T. R., Gunn, J. E., Hennessy, G. S., Knapp, G. R., Leggett, S. K., Munn, J. A., Pier, J. R., Rockosi, C. M., Schneider, D. P., Strauss, M. A., Yanny, B., Brinkmann, J., Csabai, I., Hindsley, R. B., Kent, S., Lamb, D. Q., Margon, B., McKay, T. A., Smith, J. A., Waddel, P., York, D. G., the SDSS Collaboration, Nov. 2001. Solar System Objects Observed in the Sloan Digital Sky Survey Commissioning Data. Astronomical Journal 122, $2749-2784$.

Jedicke, R., Magnier, E. A., Kaiser, N., Chambers, K. C., 2007. The next decade of Solar System discovery with Pan-STARRS. In: Valsecchi, G. B., Vokrouhlický, D., Milani, A. (Eds.), IAU Symposium. Vol. 236 of IAU Symposium. pp. 341-352.

Jedicke, R., Nesvorný, D., Whiteley, R., Ivezić, Ž., Jurić, M., May 2004. An age-colour relationship for main-belt S-complex asteroids. Nature 429, $275-277$.

Jutzi, M., Michel, P., 2014. Hypervelocity impacts on asteroids and momentum transfer i. numerical simulations using porous targets. Icarus 229, $247-253$.

Lauretta, D. S., Drake, M. J., Team, O., Dec. 2011. OSIRIS-REx - Exploration of Asteroid (101955) 1999 RQ36. AGU Fall Meeting Abstracts. 
Lazzarin, M., Marchi, S., Magrin, S., Licandro, J., Jun. 2005. Spectroscopic investigation of near-Earth objects at Telescopio Nazionale Galileo. Monthly Notices of the Royal Astronomical Society 359, 1575-1582.

Levison, H. F., Duncan, M. J., Mar. 1994. The long-term dynamical behavior of short-period comets. Icarus 108, 18-36.

Lubich, C., Walther, B., Brügmann, B., 2010. Symplectic integration of post-newtonian equations of motion with spin. Physical Review D 81 (10), 104025 .

Mainzer, A., Grav, T., Bauer, J., Masiero, J., McMillan, R. S., Cutri, R. M., Walker, R., Wright, E., Eisenhardt, P., Tholen, D. J., Spahr, T., Jedicke, R., Denneau, L., DeBaun, E., Elsbury, D., Gautier, T., Gomillion, S., Hand, E., Mo, W., Watkins, J., Wilkins, A., Bryngelson, G. L., Del Pino Molina, A., Desai, S., Gómez Camus, M., Hidalgo, S. L., Konstantopoulos, I., Larsen, J. A., Maleszewski, C., Malkan, M. A., Mauduit, J.-C., Mullan, B. L., Olszewski, E. W., Pforr, J., Saro, A., Scotti, J. V., Wasserman, L. H., Dec 2011a. NEOWISE Observations of Near-Earth Objects: Preliminary Results. Astrophysical Journal 743, 156.

Mainzer, A., Grav, T., Masiero, J., Hand, E., Bauer, J., Tholen, D., McMillan, R. S., Spahr, T., Cutri, R. M., Wright, E., Watkins, J., Mo, W., Maleszewski, C., Nov. 2011b. NEOWISE Studies of Spectrophotometrically Classified Asteroids: Preliminary Results. Astrophysical Journal 741, 90.

Marchi, S., De Sanctis, M. C., Lazzarin, M., Magrin, S., Oct. 2010. On the 
Puzzle of Space Weathering Alteration of Basaltic Asteroids. Astrophysical Journal 721, L172-L176.

Marchi, S., Paolicchi, P., Richardson, D. C., Mar. 2012. Collisional evolution and reddening of asteroid surfaces - I. The problem of conflicting timescales and the role of size-dependent effects. Monthly No 421, 2-8.

Marsset, M., Vernazza, P., Gourgeot, F., Dumas, C., Birlan, M., Lamy, P., Binzel, R. P., Aug. 2014. Similar origin for low- and high-albedo Jovian Trojans and Hilda asteroids? Astronomy and Astrophysics 568, L7.

McSween, H. Y., Lauretta, D. S., Leshin, L. A., 2006. Meteorites and the Timing, Mechanisms, and Conditions of Terrestrial Planet Accretion and Early Differentiation. Meteorites and the Early Solar System II, 53-66.

Michel, P., 1997. Effects of linear secular resonances in the region of semimajor axes smaller than 2 AU\}. Icarus 129 (2), $348-366$.

Michel, P., Migliorini, F., Morbidelli, A., Zappalà, V., Jun. 2000. The Population of Mars-Crossers: Classification and Dynamical Evolution. Icarus $145,332-347$.

Milani, A., Chesley, S. R., Valsecchi, G. B., Aug. 2000. Asteroid close encounters with Earth: risk assessment. Planetary and Space Science 48, 945-954.

Milani, A., Gronchi, G., Farnocchia, D., Knežević, Z., Jedicke, R., Denneau, L., Pierfederici, F., 2008. Topocentric orbit determination: algorithms for the next generation surveys. Icarus 195 (1), 474-492. 
Morbidelli, A., Bottke, Jr., W. F., Froeschlé, C., Michel, P., 2002. Origin and Evolution of Near-Earth Objects. Asteroids III, 409-422.

Morbidelli, A., Nesvorný, D., Jun. 1999. Numerous Weak Resonances Drive Asteroids toward Terrestrial Planets Orbits. Icarus 139, 295-308.

Mueller, M., Delbo, M., Hora, J. L., Trilling, D. E., Bhattacharya, B., Bottke, W. F., Chesley, S., Emery, J. P., Fazio, G., Harris, A. W., Mainzer, A., Mommert, M., Penprase, B., Smith, H. A., Spahr, T. B., Stansberry, J. A., Thomas, C. A., Apr. 2011. ExploreNEOs. III. Physical Characterization of 65 Potential Spacecraft Target Asteroids. Astronomical Journal 141, 109.

Murdoch, N., Abell, P., Carnelli, I., Carry, B., Cheng, A., Drolshagen, G., Fontaine, M., Galvez, A., Koschny, D., Kueppers, M., Michel, P., Reed, C., Ulamec, S., 2012. Asteroid impact \& deflection assessment (aida) mission. Tech. rep., European Space Agency.

Nakamura, T., Noguchi, T., Tanaka, M., Zolensky, M. E., Kimura, M., Tsuchiyama, A., Nakato, A., Ogami, T., Ishida, H., Uesugi, M., Yada, T., Shirai, K., Fujimura, A., Okazaki, R., Sandford, S. A., Ishibashi, Y., Abe, M., Okada, T., Ueno, M., Mukai, T., Yoshikawa, M., Kawaguchi, J., Aug 2011. Itokawa Dust Particles: A Direct Link Between S-Type Asteroids and Ordinary Chondrites. Science 333, 1113-1115.

Nesvorny, D., 2012. Nesvorny hem asteroid families v2.0. NASA Planetary Data System, eAR-A-VARGBDET-5-NESVORNYFAM-V2.0.

Nesvorný, D., Bottke, W. F., Vokrouhlický, D., Chapman, C. R., Rafkin, S., 
Oct. 2010. Do planetary encounters reset surfaces of near Earth asteroids? Icarus 209, 510-519.

Nesvorný, D., Jedicke, R., Whiteley, R. J., Ivezić, Ž., Jan. 2005. Evidence for asteroid space weathering from the Sloan Digital Sky Survey. Icarus $173,132-152$.

Ostro, S. J., Hudson, R. S., Benner, L. A. M., Giorgini, J. D., Magri, C., Margot, J. L., Nolan, M. C., 2002. Asteroid Radar Astronomy. Asteroids III, 151-168.

Parker, A., Ivezić, Ž., Jurić, M., Lupton, R., Sekora, M. D., Kowalski, A., Nov 2008. The size distributions of asteroid families in the SDSS Moving Object Catalog 4. Icarus 198, 138-155.

Perko, H. A., Nelson, J. D., Sadeh, W. Z., 2001. Surface cleanliness effect on lunar soil shear strength. Journal of geotechnical and geoenvironmental engineering 127 (4), 371-383.

Polishook, D., Binzel, R. P., Lockhart, M., DeMeo, F. E., Golisch, W., Bus, S. J., Gulbis, A. A. S., Nov. 2012. Spectral and spin measurement of two small and fast-rotating near-Earth asteroids. Icarus 221, 1187-1189.

Popescu, M., Birlan, M., Binzel, R., Vernazza, P., Barucci, A., Nedelcu, D. A., DeMeo, F., Fulchignoni, M., Nov. 2011. Spectral properties of eight near-Earth asteroids. Astronomy and Astrophysics 535, A15.

Pravec, P., Scheirich, P., Kušnirák, P., Šarounová, L., Mottola, S., Hahn, G., Brown, P. G., Esquerdo, G. A., Kaiser, N., Krzeminski, Z., Pray, 
D. P., Warner, B. D., Harris, A. W., Nolan, M. C., Howell, E. S., Benner, L. A. M., Margot, J.-L., Galád, A., Holliday, W., Hicks, M. D., Krugly, Y. N., Tholen, D. J., Whiteley, R. J., Marchis, F., Degraff, D. R., Grauer, A., Larson, S., Velichko, F. P., Cooney, W. R., Stephens, R., Zhu, J., Kirsch, K., Dyvig, R., Snyder, L., Reddy, V., Moore, S., Gajdoš, Š., Világi, J., Masi, G., Higgins, D., Funkhouser, G., Knight, B., Slivan, S. M., Behrend, R., Grenon, M., Burki, G., Roy, R., Demeautis, C., Matter, D., Waelchli, N., Revaz, Y., Klotz, A., Rieugné, M., Thierry, P., Cotrez, V., Brunetto, L., Kober, G., Mar 2006. Photometric survey of binary nearEarth asteroids. Icarus 181, 63-93.

Reddy, V., Dunn, T., Thomas, C., Moskovitz, N., Burbine, T., 2015. Mineralogy and Surface Composition of Asteroids. Asteroids IV, na.

Reddy, V., Nathues, A., Gaffey, M. J., Mar. 2011. First fragment of Asteroid 4 Vesta's mantle detected. Icarus 212, 175-179.

Ribeiro, A. O., Roig, F., Cañada-Assandri, M., Carvano, J. M. F., Jasmin, F. L., Alvarez-Candal, A., Gil-Hutton, R., Mar. 2014. The first confirmation of V-type asteroids among the Mars crosser population. Planetary and Space Science 92, 57-64.

Rivkin, A. S., Thomas, C. A., Trilling, D. E., Enga, M.-t., Grier, J. A., Feb. 2011. Ordinary chondrite-like colors in small Koronis family members. Icarus 211, 1294-1297.

Roig, F., Ribeiro, A. O., Gil-Hutton, R., Jun. 2008. Taxonomy of asteroid families among the Jupiter Trojans: comparison between spectroscopic 
data and the Sloan Digital Sky Survey colors. Astronomy and Astrophysics 483, 911-931.

Rudawska, R., Vaubaillon, J., Atreya, P., May 2012. Association of individual meteors with their parent bodies. Astronomy and Astrophysics 541, A2.

Sanchez, J. A., Michelsen, R., Reddy, V., Nathues, A., Jul. 2013. Surface composition and taxonomic classification of a group of near-Earth and Mars-crossing asteroids. Icarus 225, 131-140.

Sanchez, J. A., Reddy, V., Nathues, A., Cloutis, E. A., Mann, P., Hiesinger, H., Jul. 2012. Phase reddening on near-Earth asteroids: Implications for mineralogical analysis, space weathering and taxonomic classification. Icarus 220, 36-50.

Sánchez, P., Scheeres, D. J., May 2014. The strength of regolith and rubble pile asteroids. Meteoritics and Planetary Science 49, 788-811.

Sasaki, S., Nakamura, K., Hamabe, Y., Kurahashi, E., Hiroi, T., Mar. 2001. Production of iron nanoparticles by laser irradiation in a simulation of lunar-like space weathering. Nature 410, 555-557.

Scheeres, D. J., Hartzell, C. M., Sánchez, P., Swift, M., Dec. 2010. Scaling forces to asteroid surfaces: The role of cohesion. Icarus 210, 968-984.

Sharma, I., Jenkins, J. T., Burns, J. A., Aug. 2006. Tidal encounters of ellipsoidal granular asteroids with planets. Icarus 183, 312-330.

Solano, E., Rodrigo, C., Pulido, R., Carry, B., Feb 2013. Precovery of near- 
Earth asteroids by a citizen-science project of the Spanish Virtual Observatory. Astronomische Nachrichten 335, 142-149.

Spoto, F., Milani, A., Farnocchia, D., Chesley, S. R., Micheli, M., Valsecchi, G. B., Perna, D., Hainaut, O., 2014. Nongravitational perturbations and virtual impactors: the case of asteroid (410777) 2009 fd. Astronomy \& Astrophysics 572, A100.

Stokes, G. H., Evans, J. B., Viggh, H. E. M., Shelly, F. C., Pearce, E. C., Nov 2000. Lincoln Near-Earth Asteroid Program (LINEAR). Icarus 148, $21-28$.

Strazzulla, G., Dotto, E., Binzel, R. P., Brunetto, R., Barucci, M. A., Blanco, A., Orofino, V., mar 2005. Spectral alteration of the Meteorite Epinal (H5) induced by heavy ion irradiation: a simulation of space weathering effects on near-Earth asteroids. Icarus 174, 31-35.

Tholen, D. J., 1984. Asteroid taxonomy from cluster analysis of Photometry. Ph.D. thesis, Arizona Univ., Tucson.

Tholen, D. J., Barucci, M. A., 1989. Asteroid taxonomy. Asteroids II, 298315.

Thomas, C. A., Binzel, R. P., Feb. 2010. Identifying meteorite source regions through near-Earth object spectroscopy. Icarus 205, 419-429.

Thomas, C. A., Rivkin, A. S., Trilling, D. E., Marie-therese Enga, Grier, J. A., Mar. 2011. Space weathering of small Koronis family members. Icarus 212, 158-166. 
Thomas, C. A., Trilling, D. E., Rivkin, A. S., May 2012. Space weathering of small Koronis family asteroids in the SDSS Moving Object Catalog. Icarus 219, 505-507.

Vernazza, P., Binzel, R. P., Rossi, A., Fulchignoni, M., Birlan, M., Apr. 2009. Solar wind as the origin of rapid reddening of asteroid surfaces. Nature 458, 993-995.

Vernazza, P., Binzel, R. P., Thomas, C. A., DeMeo, F. E., Bus, S. J., Rivkin, A. S., Tokunaga, A. T., Aug. 2008. Compositional differences between meteorites and near-Earth asteroids. Nature 454, 858-860.

Vernazza, P., Delbo, M., King, P. L., Izawa, M. R. M., Olofsson, J., Lamy, P., Cipriani, F., Binzel, R. P., Marchis, F., Merín, B., Tamanai, A., Nov. 2012. High surface porosity as the origin of emissivity features in asteroid spectra. Icarus 221, 1162-1172.

Veverka, J., Robinson, M., Thomas, P., Murchie, S., Bell, J. F., Izenberg, N., Chapman, C., Harch, A., Bell, M., Carcich, B., Cheng, A., Clark, B., Domingue, D., Dunham, D., Farquhar, R., Gaffey, M. J., Hawkins, E., Joseph, J., Kirk, R., Li, H., Lucey, P., Malin, M., Martin, P., McFadden, L., Merline, W. J., Miller, J. K., Owen, W. M., Peterson, C., Prockter, L., Warren, J., Wellnitz, D., Williams, B. G., Yeomans, D. K., Sep. 2000. NEAR at Eros: Imaging and Spectral Results. Science 289, 2088-2097.

Wetherill, G. W., Jan. 1979. Steady state populations of Apollo-Amor objects. Icarus 37, 96-112. 
Willman, M., Jedicke, R., Jan. 2011. Asteroid age distributions determined by space weathering and collisional evolution models. Icarus 211, 504-510.

Willman, M., Jedicke, R., Moskovitz, N., Nesvorný, D., Vokrouhlický, D., Mothé-Diniz, T., Aug. 2010. Using the youngest asteroid clusters to constrain the space weathering and gardening rate on S-complex asteroids. Icarus 208, 758-772.

Willman, M., Jedicke, R., Nesvorný, D., Moskovitz, N., Ivezić, Ž., Fevig, R., Jun. 2008. Redetermination of the space weathering rate using spectra of Iannini asteroid family members. Icarus 195, 663-673.

Wisdom, J., Oct. 1983. Chaotic behavior and the origin of the $3 / 1$ Kirkwood gap. Icarus 56, 51-74.

Yang, B., Jewitt, D., Jul. 2007. Spectroscopic Search for Water Ice on Jovian Trojan Asteroids. Astronomical Journal 134, 223-228.

Yang, B., Jewitt, D., Mar. 2011. A Near-infrared Search for Silicates in Jovian Trojan Asteroids. Astronomical Journal 141, 95.

Yano, H., Yoshikawa, M., Yano, H., Tsuda, Y., Nakazawa, S., Mimamino, H., Terui, F., Saiki, T., Nishiyama, K., Kubota, T., Okada, T., Morimoto, M. Y., Ogawa, N., Okamoto, C., Takagi, Y., Tachibana, S., Nakamura, R., Hirata, N., Demura, H., 2010. Hayabusa's follow-on mission for surface and sub-surface sample return from a C-type NEO. In: 38th COSPAR Scientific Assembly. Vol. 38 of COSPAR Meeting. p. 635.

Ye, Q.-z., Feb. 2011. BVRI Photometry of 53 Unusual Asteroids. Astronomical Journal 141, 32. 
Yoshida, H., 1990. Construction of higher order symplectic integrators. Physics Letters A 150 (5), 262-268.

Yu, Y., Richardson, D. C., Michel, P., Schwartz, S. R., Ballouz, R.-L., Nov. 2014. Numerical predictions of surface effects during the 2029 close approach of Asteroid 99942 Apophis. Icarus 242, 82-96.

\section{Appendix A. Forces model}

In the following we will briefly discuss the force models used in equations (2) and (4).

\section{Appendix A.1. Self gravity of the asteroid}

On the surface of the asteroid a particle of mass $\mu$ will experience the following gravitational pull towards the asteroid's center of mass:

$$
F_{g a}=\mathcal{G} \mu m / R^{2}
$$

where $\mathcal{G}$ is the gravitational constant, $m$ the mass and $R$ the local radius of the asteroid.

\section{Appendix A.2. Forces due to the Rotating Frame}

In a rotating frame of reference, surface particles are subject to a centrifugal force $F_{c f}$. It will suffice to consider principle axis rotation as we are primarily interested in best and worst case scenarios. Therefore, the particle feeling the maximum centrifugal acceleration then is located farthest from the axis of rotation and we have

$$
F_{c f}=\mu R \omega^{2} \cos \phi
$$


Here, $\omega$ is the spin rate and $\phi$ the angle between the asteroid's spin axis and the vector $\vec{r}$ connecting the centers of gravity of the planet and the asteroid. In other words $\phi$ is the asteroids obliquity with respect to is planetocentric orbit. Consequently, $F_{c f}^{\max }=\mu R^{\max } \omega_{s b}^{2}$. This corresponds to a particle on the equator of a spherical asteroid with maximum radius $R^{\max }$ and a rotation rate close to the spin barrier $\omega_{s b}$. As we assume that the particles are at rest on the surface, no Coriolis forces will have to be accounted for.

\section{Appendix A.3. Tidal Acceleration}

Asteroid that have close encounters with other massive bodies will experience tidal forces. Towards an asteroid's surface the tidal acceleration becomes stronger compared to volume elements that are closer to the center of gravity. Consequently surface particles are pulled away more easily than say boulders close to the center of the asteroid. A simple model for the tidal force acting on a particle with mass $\mu$ is:

$$
F_{t d}=2 R \mathcal{G} \mu M / D^{3}
$$

where $M$ is the mass of the planet and $D$ the distance between the asteroid

and the planet. In principle, there are additional tidal contributions from the sun, and the moon if the Earth is approached. Those are, however, small compared to the contribution of the Earth itself and shall, therefore, be neglected.

\section{Appendix A.4. Particle Cohesion}

The role of cohesion in rubble pile asteroids has been studied extensively over the past years (Scheeres et al. 2010; Hartzell and Scheeres 2011; 
Sánchez and Scheeres 2014). It is currently understood that particle cohesion plays and important role in determining limits for rubble-pile asteroid failures, fission, and for the survival of contact binaries. The standard cohesion model as presented in Hartzell and Scheeres (2011) is based on van der Waals interaction in granular materials. The inter particle force can be modeled as:

$$
F_{c o}=C S^{2} \sigma
$$

where $C=5.14 \times 10^{-2} \mathrm{~kg} / \mathrm{s}^{2}$ is a material constant related to the Hamaker constant, $S \sim 1$ is the so-called cleanliness ratio, a qualitative factor indicating the impurity of granular surface coatings and $\sigma$ is the radius of the particles on the asteroid's surface. For a detailed discussion of equation A.4 see e.g. Hartzell and Scheeres (2011). As it is harder to free larger particles or rocks from the surface, we will increase the particle radius that shall be lifted against cohesion. Equation (A.4) illustrates that the larger the particles and the cleaner the surface, the larger the cohesive force. Let us now determine the approximate size of the largest particles that will be relevant for our resurfacing considerations. For this purpose we assume an $N \propto(2 \sigma)^{-3}$ distribution of the number of particles $N$ on the asteroid's surface. Using equation (36) in Sánchez and Scheeres (2014) we can calculate the particle diameter up to which smaller particles cover $99 \%$ of the surface. This is $\sigma=100 r_{0}$, where we furthermore assume that $r_{0}$ is of the order of the smallest particle radii that could be detected on Itokawa $\left(r_{0} \simeq 10^{-6} \mathrm{~m}\right)$. Hence, practically all of the asteroid's surface is covered by particles smaller than 0.1 millimeters. 